\title{
Estimating Oceanic Turbulence Dissipation from Seismic Images
}

\author{
W. Steven Holbrook, ${ }^{*}$ Ilker Fer, ${ }^{+}$Raymond W. Schmitt, ${ }^{\#}$ Daniel Lizarralde, ${ }^{@}$ \\ JODY M. KLYMAK, ${ }^{\&}$ L. CODY HELFRICH $,{ }^{*},++$ AND ROBERT KUBICHEK $* *$ \\ * Department of Geology and Geophysics, University of Wyoming, Laramie, Wyoming \\ ${ }^{+}$Geophysical Institute, University of Bergen, Bergen, Norway \\ \# Department of Physical Oceanography, Woods Hole Oceanographic Institution, Woods Hole, Massachusetts \\ @ Department of Geology and Geophysics, Woods Hole Oceanographic Institution, Woods Hole, Massachusetts \\ \& School of Earth and Ocean Sciences, University of Victoria, Victoria, British Columbia, Canada \\ ** Department of Electrical and Computer Engineering, University of Wyoming, Laramie, Wyoming
}

(Manuscript received 11 July 2012, in final form 10 January 2013)

\begin{abstract}
Seismic images of oceanic thermohaline finestructure record vertical displacements from internal waves and turbulence over large sections at unprecedented horizontal resolution. Where reflections follow isopycnals, their displacements can be used to estimate levels of turbulence dissipation, by applying the Klymak-Moum slope spectrum method. However, many issues must be considered when using seismic images for estimating turbulence dissipation, especially sources of random and harmonic noise. This study examines the utility of seismic images for estimating turbulence dissipation in the ocean, using synthetic modeling and data from two field surveys, from the South China Sea and the eastern Pacific Ocean, including the first comparison of turbulence estimates from seismic images and from vertical shear. Realistic synthetic models that mimic the spectral characteristics of internal waves and turbulence show that reflector slope spectra accurately reproduce isopycnal slope spectra out to horizontal wavenumbers of $\sim 0.04 \mathrm{cpm}$, corresponding to horizontal wavelengths of $25 \mathrm{~m}$. Using seismic reflector slope spectra requires recognition and suppression of shot-generated harmonic noise and restriction of data to frequency bands with signal-to-noise ratios greater than about 4. Calculation of slope spectra directly from Fourier transforms of the seismic data is necessary to determine the suitability of a particular dataset to turbulence estimation from reflector slope spectra. Turbulence dissipation estimated from seismic reflector displacements compares well to those from 10-m shear determined by coincident expendable current profiler (XCP) data, demonstrating that seismic images can produce reliable estimates of turbulence dissipation in the ocean, provided that random noise is minimal and harmonic noise is removed.
\end{abstract}

\section{Introduction}

Turbulence in the ocean is an important mechanism for transporting momentum and heat and dispersing nutrients, pollutants, and small organisms. The principal measure of oceanic turbulence is the dissipation rate of turbulence kinetic energy $\varepsilon$ per unit mass, which involves work against buoyancy and the loss of mechanical energy to heat energy through turbulent motions and

${ }^{++}$Current affiliation: BP America, Houston, Texas.

Corresponding author address: W. Steven Holbrook, Dept. of Geology and Geophysics, University of Wyoming, 1000 E. University Ave., Laramie, WY 82072.

E-mail: steveh@uwyo.edu has units of watts per kilogram. Dissipation can be related to vertical eddy diffusivity $K_{\rho}$ (Osborn 1980). In the ocean's interior, turbulence is primarily driven by breaking of internal waves and hydraulic phenomena (Wunsch and Ferrari 2004) and is largely responsible for the vertical mixing that maintains the global overturning circulation (e.g., Munk and Wunsch 1998). To close global mixing budgets, a global average $K_{\rho}$ of $10^{-4} \mathrm{~m}^{2} \mathrm{~s}^{-1}$ is required-yet studies of ocean mixing in the openocean thermocline tend to find values an order of magnitude too low (e.g., Ledwell et al. 1993). This mismatch suggests that ocean mixing may be largely controlled by "hot spots" of mixing controlled by enhanced atmospheric forcing and the impingement of tidal and deepreaching currents on topography, including seamounts (Lueck and Mudge 1997; Toole et al. 1997), midocean ridges (St. Laurent and Garrett 2002), island chains 
(Rudnick et al. 2003), and rough seafloor (Naveira Garabato et al. 2004; Polzin et al. 1997). Despite its central importance to ocean mixing, turbulence is challenging to quantify and characterize over large sections of the ocean because it is patchy in space and intermittent in time (Ivey et al. 2008). In this paper we examine the efficacy of a new method for characterizing turbulence: seismic reflection images of oceanic fine structure.

Seismic oceanography (SO) is a relatively new approach to imaging oceanic thermohaline fine structure with low-frequency $(\sim 20-200 \mathrm{~Hz})$ acoustic reflections collected during marine seismic reflection profiling (Holbrook et al. 2003). The physical basis for seismic oceanography has been well established by several studies that combined seismic surveys with simultaneous in situ measurements of temperature, salinity, and density (Nakamura et al. 2006; Nandi et al. 2004; Sallarès et al. 2009): weak, but clear, reflections from within the water column come from vertical changes in (primarily) sound speed and (secondarily) density. Over the past few years, numerous studies have demonstrated the ability of seismic imaging to detect and map major features in the ocean, including fronts (Holbrook et al. 2003; Mirshak et al. 2010; Sheen et al. 2009), internal waves (Holbrook and Fer 2005; Krahmann et al. 2008), eddies and warm-core rings (Biescas et al. 2008; Ruddick et al. 2009; Yamashita et al. 2011), thermohaline staircases (Biescas et al. 2010; Fer et al. 2010), lee waves (Eakin et al. 2011), and internal tide beams (Holbrook et al. 2009). SO has several unique capabilities, including the ability to image fine structure over large sections of the ocean and to full ocean depth, provided that sufficient fine structure is present to produce reflections. But perhaps the greatest advantage provided by seismic reflection images in the ocean is the dense lateral sampling of the data: typical seismic images provide seismic traces (vertical profiles of reflected amplitude) at $6.25-\mathrm{m}$ spacing. [We note that horizontal resolution, as opposed to sampling, in migrated seismic reflection images is conservatively estimated as half the effective seismic wavelength (Claerbout and Fomel 2006), or $\sim 20 \mathrm{~m}$ for a sound speed of $1500 \mathrm{~m} \mathrm{~s}^{-1}$ and a dominant frequency of $40 \mathrm{~Hz}$.] This horizontal sampling is unique among oceanographic measurement techniques, apart from horizontally towed instruments (e.g., Katz 1975; Moum et al. 2002), which are typically restricted to a very limited depth range.

A major challenge of seismic oceanography is to extract from the seismic images reliable quantitative information about the internal wave and turbulence fields. Holbrook and Fer (2005) first showed that horizontal wavenumber $k_{x}$ spectra of tracked seismic reflectors mimic the Garrett-Munk spectrum and thus carry information about the internal wave field. Subsequent work has corroborated the sensitivity of the seismic method to internal waves (Krahmann et al. 2008), although wavenumber spectra may be distorted in the presence of strong currents (Vsemirnova et al. 2009). At the highest wavenumbers accessible to seismic imaging, turbulence may also be detectable: Holbrook and Fer (2005) pointed out an apparent $k_{x}^{-5 / 3}$ subrange in their spectra and speculated that this might indicate turbulence dissipation; this was supported using slope spectra by Klymak and Moum (2007b). Sheen et al. (2009) applied Klymak and Moum's slope spectra method to seismic images and interpreted spatial variations in turbulence dissipation on a seismic section in the Southern Ocean. The basis of this approach is the model proposed by Klymak and Moum (2007a,b, hereinafter KM07) relating horizontal slope spectra to turbulence dissipation via a Batchelor spectrum. KM07 showed that turbulence can have a signature that extends to surprisingly long horizontal wavelengths, up to several hundred meters in the case of high turbulence levels-wavelengths easily captured by seismic images.

Here we assess the use of horizontal wavenumber information to extract estimates of turbulence dissipation from seismic reflection images. Our study includes full-wavefield synthetic tests of the sensitivity of seismic data in the $k_{x}$ domain, an analysis of the effects of random and shot-generated noise, and the first corroboration of seismically derived turbulence estimates against measurements from collocated, in situ oceanographic data. Using new approaches to estimate horizontal wavenumber content directly from the seismic data and via tracked reflections, we show that seismically derived horizontal slope spectra often show a clear turbulence slope and provide estimates of vertical diffusivity in the expected range $\left(\sim 5 \times 10^{-4} \mathrm{~m}^{2} \mathrm{~s}^{-1}\right)$, provided that harmonic noise is minimal and that nonrandom noise is removed. A comparison of $K_{\rho}$ derived from seismic data to that derived from 10-m shear measured on coincident expendable current profilers (XCPs) shows good agreement. These results demonstrate that seismic oceanography can provide a useful tool to remotely quantify mixing processes in the ocean.

\section{Background}

Recent work by KM07 on horizontal thermistor tows shows that, in contrast to vertical strain spectra, horizontal isopycnal slope spectra are sensitive to turbulence at surprisingly low horizontal wavenumbers. The slope spectra are formed from the horizontal gradient of vertical isopycnal displacement $\zeta_{x}$ by multiplying the vertical isopycnal displacement spectra $\phi_{\zeta}$ by $\left(2 \pi k_{x}\right)^{2}$, 
A.

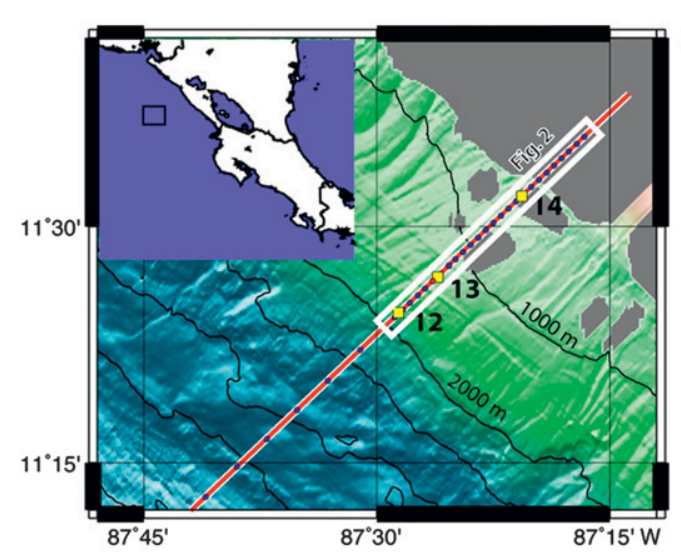

B.

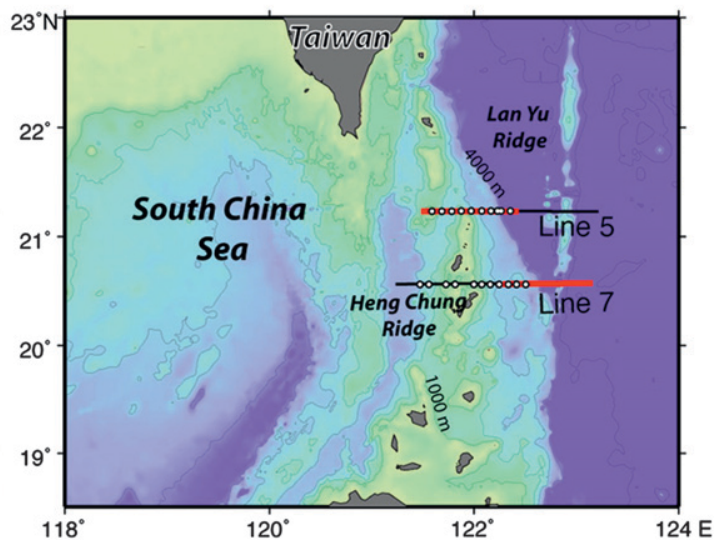

FIG. 1. Location maps for seismic data shown in this paper; bathymetry is contoured at $1000 \mathrm{~m}$. (a) Location map of line SO-1, offshore Nicaragua, showing locations of XBTs (solid circles) and XCPs (yellow squares). (b) Location map of lines acquired in Luzon Straits of South China Sea, showing locations of XBTs (open circles). Data from parts of lines 5 and 7 (denoted by red lines) are shown in this paper.

where $k_{x}$ is the horizontal wavenumber. The $k_{x}$ slope spectrum at wavenumbers between about $10^{-3}$ and $10^{\circ} \mathrm{cpm}$ contains two distinct subranges: a "red" subrange at lower $k_{x}$ dominated by internal waves and a "blue" subrange at higher $k_{x}$ dominated by turbulence dissipation. Following the Garrett-Munk (GM) internal wave formalism, using the Garrett and Munk (1975, hereinafter GM75) model, the spectral levels in the internal wave subrange decrease proportionately to frequency content. KM07 show spectral slopes in the internal wave regime between -1 and 0 , and suggest that spectra are fit to -0.5 slope, consistent with GM75:

$$
\varnothing_{\zeta_{x}}^{\mathrm{IW}}\left(k_{x}\right) \approx \phi_{0} k_{x}^{-0.5}
$$

where $\varnothing_{\zeta_{x}}^{\mathrm{IW}}$ is the slope spectrum in the internal wave subrange, and $\phi_{0}$ is an amplitude that depends on stratification, energy level, and the internal wave field's frequency content.

In the turbulence subrange, the spectral level depends on the level of dissipation (and therefore $K_{\rho}$ ), according to Eq. (12) of Klymak and Moum (2007b):

$$
\begin{aligned}
\varnothing_{\zeta_{x}}^{\text {Turb }}\left(k_{x}\right)= & 4 \pi \frac{\Gamma \varepsilon}{N_{0}^{2}}\left[C_{T^{-1 / 3}}\left(2 \pi k_{x}\right)^{1 / 3}\right. \\
& \left.+q \nu^{1 / 2} \varepsilon^{-1 / 2}\left(2 \pi k_{x}\right)\right]\left(\mathrm{cpm}^{-1}\right),
\end{aligned}
$$

where $\varnothing_{\zeta_{x}}^{\text {Turb }}$ is the slope spectrum in the turbulence subrange, $\varepsilon$ is the turbulence dissipation rate, $\Gamma$ is an empirical constant set to 0.2 (Osborn 1980), $C_{T}$ is a constant set to $0.4, N_{0}$ is the mean buoyancy frequency, $q$ is an empirical constant set to 2.3 , and $v$ is the viscosity of seawater. The right-hand side of (2) contains two terms that describe turbulence: an inertial-convective subrange, which is dependent only on dissipation and wavenumber, and an inertial-diffusive subrange, which also depends on the viscosity of seawater. At the wavenumbers relevant to seismic imaging, the inertialconvective subrange dominates, producing slope spectra that are proportional to $k_{x}{ }^{1 / 3}$ (Klymak and Moum 2007b), according to the second term in Eq. (2), as will be seen below.

The KM07 model provides a sound basis for interpreting $k_{x}$ slope spectra derived from seismic reflection images. The turbulence subrange extends to surprisingly large horizontal wavelengths $(>100 \mathrm{~m})$; these are spatial scales easily resolved in reflection images of fine structure. Here, we investigate the applicability of the KM07 model to seismic images, with special attention to pitfalls in seismic acquisition and processing that must be avoided in order to produce reliable slope spectra from seismic images.

\section{Methods}

\section{a. Seismic data acquisition and processing}

The field seismic data analyzed here come from two seismic surveys, both conducted by the R/V Marcus Langseth, a specialized seismic vessel. Data from offshore Nicaragua (Fig. 1) were acquired in March 2008 using a 6-km-long, 480-channel hydrophone streamer, with hydrophone groups spaced at $12.5 \mathrm{~m}$. The seismic source was an 18-element, 3300-in. ${ }^{3}$ air gun array. Line SO-1 was acquired as a dedicated seismic oceanography 
line, with two modifications to the acquisition plan designed to enhance water-column reflection imaging: 1 ) a shot spacing of $25 \mathrm{~m}$, which minimizes harmonic noise, as will be discussed below; and 2) a sample rate of $0.001 \mathrm{~s}$, giving a Nyquist frequency of $500 \mathrm{~Hz}$. In addition, expendable instruments [ 23 expendable bathythermographs (XBTs), three XCPs, and one expendable conductivitytemperature-depth (XCTD) probe] were deployed along the line at $\sim 1-\mathrm{km}$ intervals during seismic acquisition to record ocean temperature, salinity, and current data.

The South China Sea data (Fig. 1) were acquired in summer 2009 using a 6-km-long, 465-channel hydrophone streamer and a 36-element, 6600-in. ${ }^{3}$ air gun array as the sound source. We present data from two lines in this paper; line 5 was acquired using a $50-\mathrm{m}$ shot spacing and line 7 using a 150-m shot spacing. Coincident temperature data were acquired on both lines using XBTs, which were deployed during seismic shooting at spacing of approximately $10 \mathrm{~km}$.

Seismic data acquired here were processed through a standard processing flow, including velocity analysis, common midpoint stacking, and poststack migration. Migrated stacks of the lines discussed here are shown in Fig. 2. A complete review of seismic processing methods is beyond the scope of this paper, but useful summaries can be found in standard textbooks (e.g., Sheriff and Geldart 1995; Yilmaz 1987). Ruddick et al. (2009) summarize the basics of seismic processing for an oceanographic audience. Velocity moveout analyses were conducted by hand in order to provide the best possible images (Fortin and Holbrook 2009). For the purposes of creating horizontal wavenumber spectra, particular attention must be paid to frequency-domain filters. Prior to stacking, we applied a minimum-phase, trapezoidal bandpass filter with corner frequencies of 20-30-80 $90 \mathrm{~Hz}$. As will be discussed further below, we found that it is important to apply the filters prior to stacking and migration in order to minimize random noise at high $k_{x}$. Given the tow depth of the guns and streamer at $6 \mathrm{~m}$, the first "ghost notch" in the frequency spectrum occurs at $125 \mathrm{~Hz}$, beyond our passband. No lateral smoothing of the data was performed, as this would strongly affect $k_{x}$ spectra. Amplitudes were corrected for spherical divergence. Depths were calculated by converting twoway travel times to depth assuming a constant velocity of $1500 \mathrm{~m} \mathrm{~s}^{-1}$.

\section{b. Calculation of synthetic seismograms}

To test whether seismic reflection images can faithfully reproduce isopycnal displacements in a turbulent ocean, we simulated seismic wave propagation through a synthetic ocean sound speed model containing characteristics of internal waves and turbulence. The model

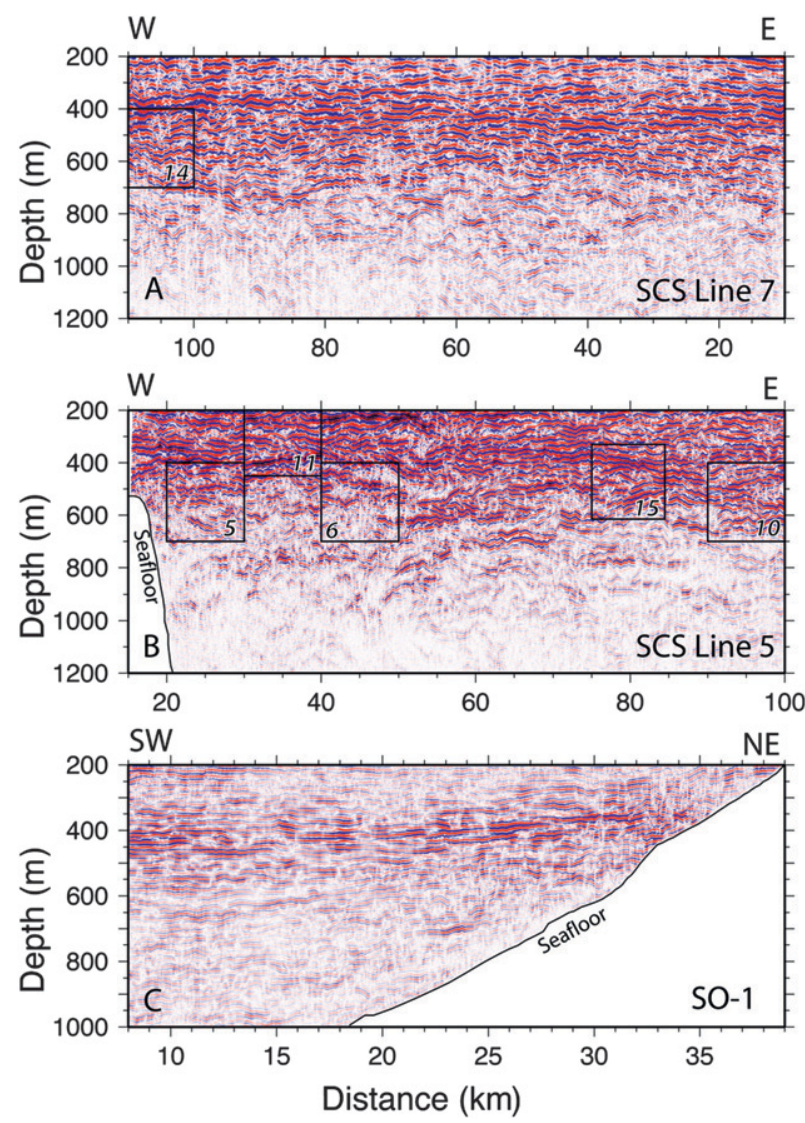

FIG. 2. Migrated stacks of seismic cross sections used in this paper; horizontal axis is distance along ship track. Data are plotted so that positive-amplitude returns (peaks) are blue and negativeamplitude returns (troughs) are red. Boxes show portions of seismic sections shown in zooms in later figures; numbers in boxes refer to figure number. (a) South China Sea, line 7. (b) South China Sea, line 5. (c) Offshore Nicaragua, line SO-1.

does not include the physics of internal wave generation or propagation or of turbulence; rather, it merely creates displacements of isotachs that mimic the spectral characteristics of internal waves and turbulence according to the KM07 model. While full physical modeling of these processes at the scales appropriate to seismic waveform modeling would be worthwhile, the present simulation is sufficient to determine whether seismic data at typical wavelengths are capable of faithfully capturing isotach (and therefore isopycnal) displacements with the length scales characteristic of internal waves and turbulence. The model includes internal wave displacements for a spectrum with Garrett-Munk frequency content at 3 times the GM energy; turbulent displacements are created by simulating the inertialconvective $\left(k_{x}^{-5 / 3}\right)$ subrange with a user-defined scale factor that can set turbulent energy independently of internal wave energy. 

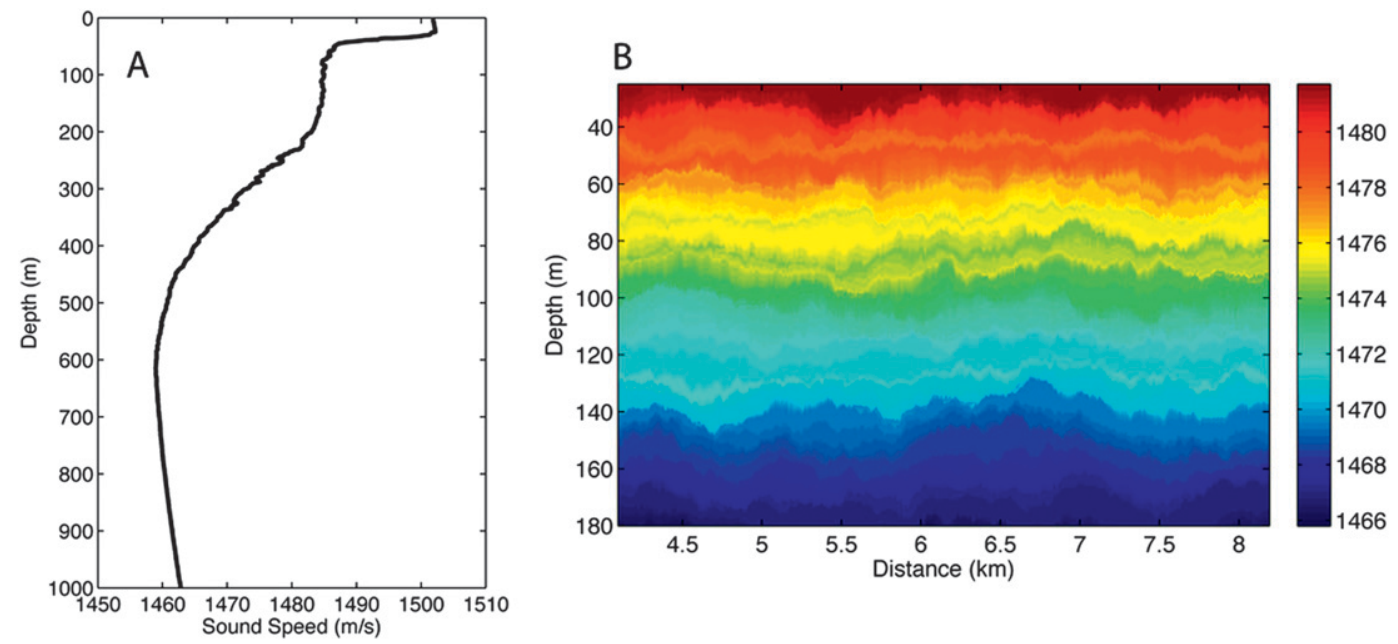

FIG. 3. (a) Sound speed profile from XCTD probe 51 in the Norwegian Sea (Nandi et al. 2004), used to create sound speed section for calculation of synthetic seismograms. (b) Sound speed section created by starting with sound speed profile from profile in (a) over depths of $202-400 \mathrm{~m}$ and applying displacements that simulate the spectral characteristics of internal waves and turbulence, for moderate turbulence (corresponding to a diffusivity of $\sim 2 \times 10^{-4}$ $\left.\mathrm{m}^{2} \mathrm{~s}^{-1}\right)$. Color scale shows sound speed $\left(\mathrm{m} \mathrm{s}^{-1}\right)$.

The sound speed model comes from an XCTD probe deployed during a seismic survey in the Norwegian Sea (and discussed by Nandi et al. 2004), shown in Fig. 3. The purpose of the synthetic modeling is to show that, for typical acquisition parameters and geometry, seismic images are capable of resolving the horizontal wavenumber characteristics associated with oceanic turbulence. A site-specific initial sound speed structure thus does not affect the generality of the results. Weak sound speed inversions corresponding to thermohaline intrusions are visible in the depth range of $\sim 100-500 \mathrm{~m}$. To limit the computational intensity of the model, we limited the model to a region about $200 \mathrm{~m}$ in thickness centered on $300-\mathrm{m}$ depth. We created an 8-km-long synthetic sound speed model by repeating the sound speed profile of Fig. 3a at 8-m intervals across the model space, then displacing sound speed values by a vertical distance specified by the KM07 model at each model grid point, over a range of turbulence values, and interpolating the values onto a constant depth interval of $1 \mathrm{~m}$. (We note that our methodology does not take into account slight differences between isotherms and isopycnals that may occur for thermohaline intrusions.)

The synthetic seismic section was created by propagating a wave field through the synthetic wave-speed model (Fig. 3b). We used a pseudospectral algorithm (Kosloff and Baysal 1982) to propagate the wave field generated by "exploding reflector" sources (Loewenthal et al. 1976), which are sources located at every point in the model, each initiating at time zero and each with an amplitude equal to the local seismic impedance contrast.
The simulated seismic wave field, as extracted from the surface nodes of the model, replicates a stacked, zerooffset reflection section and includes lateral seismic wave propagation effects and internal multiple reflections. We then migrated this synthetic, zero-offset seismic section to place reflectors in their proper spatial positions ( $x$ and $z$ ) using a one-dimensional, depthvarying, wave-speed profile defined by the average seismic wave speed at each depth (Gazdag 1978). This modeling approach represents a good balance between overly simplistic convolution methods and a fully realistic simulation of field seismic data. The latter would require simulation of individual shots across the $24-\mathrm{km}$ extent of the model, prestack processing, and stacking. A full-blown synthetic test of this type would be useful for exploring the impact of lateral smearing that may occur through the stacking process. However, the present work aims to explore the potential of utilizing the wavenumber spectra of fine structure intrinsically recorded in perfectly processed reflection seismic data. A comparison of the migrated seismic section to the isopycnals shows that reflector displacements track isopycnal displacements well (Fig. 4), thus demonstrating the efficacy of the seismic reflection method to produce estimates of isopycnal displacement.

\section{c. Horizontal slope spectra of seismic data}

Previous efforts to extract horizontal wavenumber information from seismic images of oceanic fine structure have used tracked seismic reflectors to do so (Holbrook and Fer 2005; Krahmann et al. 2008; Sheen 


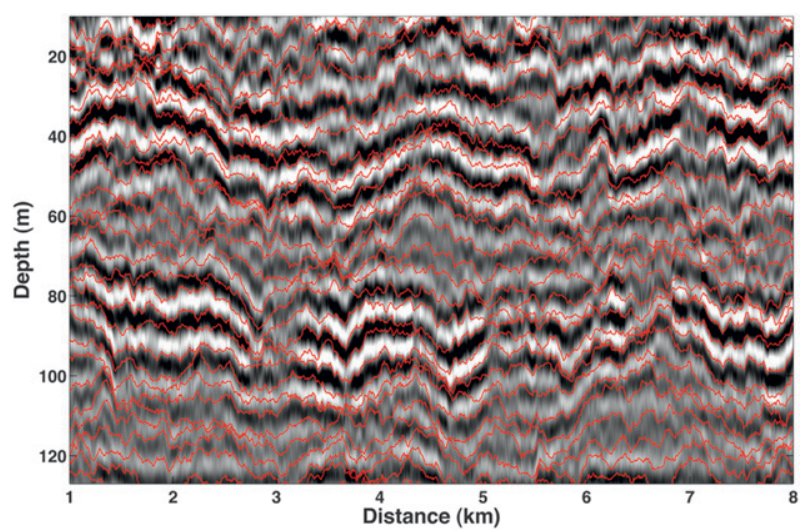

FIG. 4. Synthetic seismic section created by simulating seismic propagation through the sound speed model shown in Fig. 3. Seismic section is plotted so that positive-amplitude returns (peaks) are black and negative-amplitude returns (troughs) are white. Red lines are isopycnals from sound speed model of Fig. 3. Note the good correspondence between the displacements of reflections and isopycnals.

et al. 2009). While this approach is necessary in order to estimate vertical displacement spectra, it is indirect, in that it relies on an extracted or interpreted quantity (tracked reflectors). A useful way to more directly assess the horizontal wavenumber content of seismic reflection data - and in particular to identify noise contaminationis to calculate $k_{x}$ spectra directly from the seismic data, rather than from tracked horizons. We accomplish this by 1 ) calculating the $k_{x}$ spectrum (periodogram) of the seismic reflection amplitudes at each depth level of the image, 2) multiplying the $k_{x}$ spectrum by $\left(2 \pi k_{x}\right)^{2}$ to produce a slope spectrum, and then 3 ) averaging the slope spectra from each depth level to produce a mean $k_{x}$ spectrum of the given image (Fig. 5).

Horizontal slope spectra calculated directly from seismic data generally show very clear and sharp cutoffs from an internal wave subrange to a turbulence subrange. In many cases (though not all), the turbulence subrange shows slopes very close to the predicted $k_{x}{ }^{1 / 3}$ slope for turbulence dissipation (Fig. 5). The transition in spectral slope usually occurs between $k_{x}$ values of 0.005 and $0.01 \mathrm{cpm}$ (horizontal wavelengths of 100$500 \mathrm{~m}$ ), similar to the transitions observed in isopycnal slope spectra data in KM07. The positive spectral slope seen in Fig. 5 cannot be due to random noise, which would be flat (white) in a $k_{x}$ spectrum and thus have a slope of $k_{x}^{2}$ in a slope spectrum. The observed sharp change in spectral slope in Fig. 5 provides strong circumstantial evidence that seismic data contain information about turbulence as well as internal waves. (The drop-off at high wavenumbers is due to frequency filtering of the seismic data from 30 to $80 \mathrm{~Hz}$, as will be described in detail in the following section.)
In datasets we have analyzed, the sharp change in slope of $k_{x}$ slope spectra is a nearly ubiquitous feature. Frequently, however, the observed slope of the "turbulence" subrange slightly exceeds $k_{x}^{1 / 3}$, though it is clearly not a $k_{x}^{2}$ noise slope (Fig. 6). The reasons for this are not entirely clear, though we can speculate about several possibilities. First, sources of nonrandom noise (e.g., harmonic shot-generated noise, section 3e below) may contaminate the spectra. This possibility is corroborated by the observation that tracked reflector slope spectra often have slopes closer to $k_{x}^{1 / 3}$ even when the seismic data transforms show higher slopes, as discussed further in section 3f. Second, our imaging assumes stationary targets; if strong currents are present at depth, then they will distort $k_{x}$ spectra (Vsemirnova et al. 2009). However, we consider this effect to be an unlikely source of the enhanced slopes because 1) currents in a 300-m-thick section would not be expected to be entirely in one direction and 2) we rarely see turbulence subrange slopes lower than the expected $k_{x}{ }^{1 / 3}$.

Horizontal slope spectra calculated directly from seismic data provide a profitable view of the spectral characteristics of a seismic dataset, especially the noise characteristics. Such "data transforms" should be calculated before proceeding with reflector tracking to determine whether a given seismic oceanography dataset is amenable to meaningful reflector slope spectra.

\section{d. Suppression of random noise}

Random noise affects all seismic datasets and must be taken into account when examining $k_{x}$ spectra. "White noise" is flat in $k_{x}$ spectra and therefore appears with a distinctive $k_{x}^{2}$ slope in slope spectra. The level of random noise in a dataset can be characterized by the signal-to-noise $(\mathrm{S} / \mathrm{N})$ ratio, which for any two adjacent seismograms can be defined as

$$
\frac{S}{N}=\sqrt{\frac{|c|}{|a-c|}},
$$

where $c$ is the maximum value of the cross correlation of the two traces and $a$ is the zero-lag autocorrelation value of the first trace. To estimate $\mathrm{S} / \mathrm{N}$ for a section of data, we calculate $\mathrm{S} / \mathrm{N}$ for every adjacent pair of traces using the equation above and then calculate the median $\mathrm{S} / \mathrm{N}$ value for the section.

Because seismic air gun sources are bandlimited (Fig. 7), with useful signal strength between $\sim 10$ and $150 \mathrm{~Hz}$ (the details depend on the particular source array and towing depth), S/N varies as a function of frequency in seismic datasets. The appearance of a seismic image and its $k_{x}$ characteristics depend on the frequency bands 

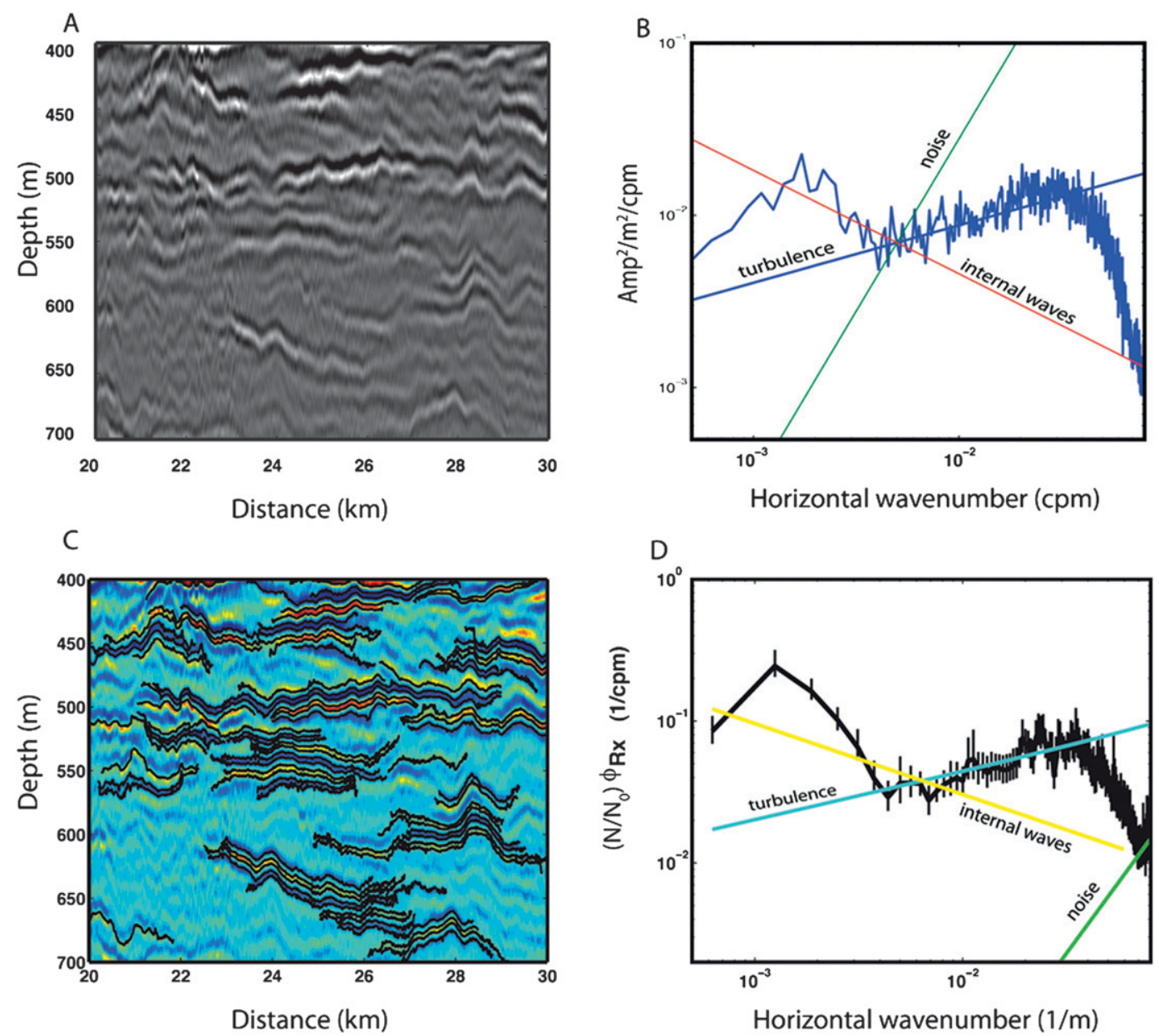

FIG. 5. (a) Zoom of seismic data from South China Sea line 5, from $x=20-30 \mathrm{~km}$ (full line is shown in Fig. 2). (b) Horizontal wavenumber $k_{x}$ spectrum calculated directly from seismic data, by calculating a 2D Fourier transform of the seismic section, multiplying by $\left(2 \pi k_{x}\right)^{2}$, and summing spectral levels at each $k_{x}$ value. "Amp" is the amplitude value of seismic data (arbitrary units). Straight lines show slopes that would be present because of turbulence, internal waves, and noise. Note clear $k_{x}^{1 / 3}$ subrange above wavenumbers of $\sim 0.005 \mathrm{cpm}$. The $\mathrm{S} / \mathrm{N}$ ratio of this section of data is 6.9. (c) Seismic image showing tracked reflections (black lines) from the same section shown in (a). (d) Slope spectrum calculated from tracked reflections using a 256-point Fourier transform. Vertical lines show 95\% confidence interval. Blue line shows turbulence fit; yellow line is internal wave subrange fit; and green line is example noise slope. Note the similarity of the reflector-based slope spectrum to the $k_{x}$ spectrum calculated from a $2 \mathrm{D}$ Fourier transform of the data (b).

contained in the image (Fig. 8). Before calculating $k_{x}$ slope spectra for a particular dataset, then, it is important to filter out frequency bands that are contaminated by random noise. To determine optimum frequency bands for horizontal wavenumber analysis for line SO-1, we applied a sequence of frequency bandpass filters to a representative panel of data (Fig. 8) and calculated $k_{x}$ slope spectra (data transforms) for each panel (Fig. 9). We used a zero-phase, trapezoidal filter; passbands are $10 \mathrm{~Hz}$ in width, with 10-Hz-wide drop-offs (e.g., the " $25 \mathrm{~Hz}$ " curve in Fig. 9 represents the result of a $20-30-\mathrm{Hz}$ passband, with a lowcut at $10 \mathrm{~Hz}$ and a highcut at $40 \mathrm{~Hz}$ ). After frequency filtering, $k_{x}$ slope spectra were calculated for each passband using the method described in the previous section. The results enable analysis of the horizontal wavenumber characteristics of different frequency components of the seismic images.

The passband analysis shows that different frequency components are sensitive to different parts of the $k_{x}$ spectrum (Fig. 9). Lower seismic frequencies carry information about internal waves in the lower horizontal wavenumbers but are relatively insensitive to higher wavenumbers and thus less informative about turbulence. Higher seismic frequencies, in contrast, are sensitive to shorter horizontal length scales and thus more sensitive to turbulence than lower frequencies, but they 
A

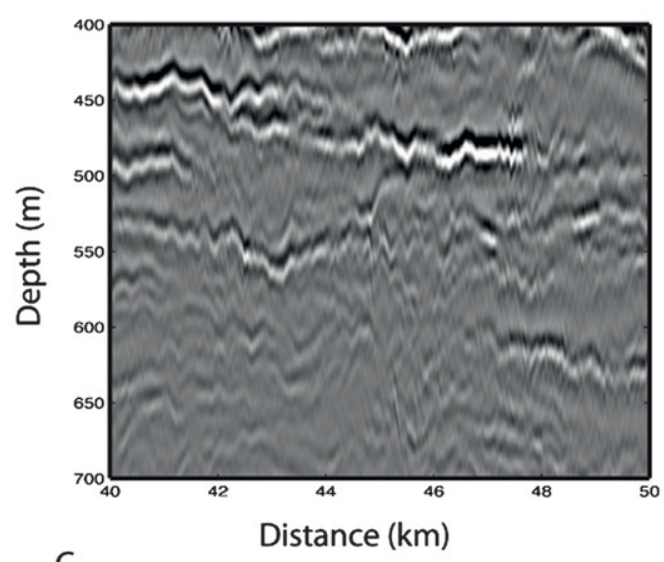

C

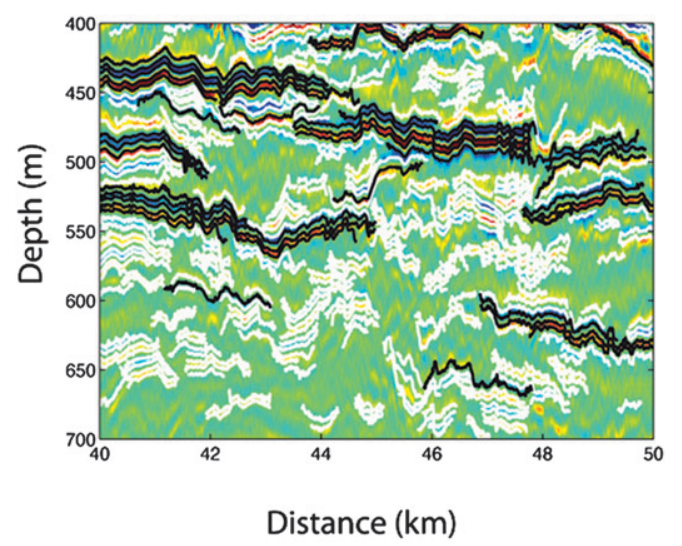

B
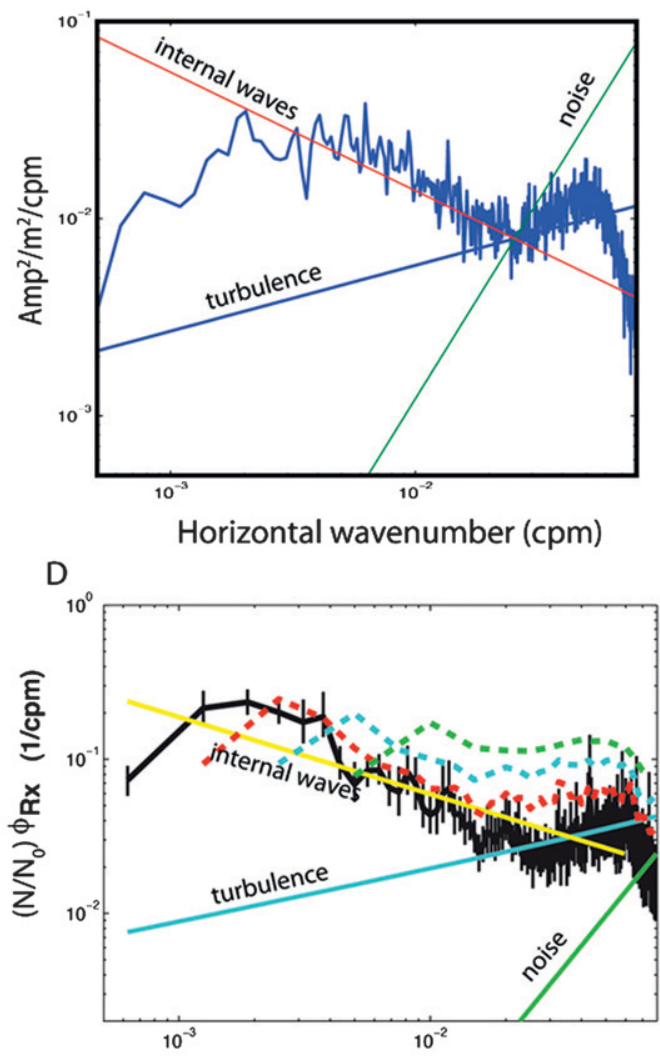

Horizontal wavenumber (cpm)

FIG. 6. (a) Zoom of seismic data from South China Sea line 5, from $x=40-50 \mathrm{~km}$. (b) Horizontal wavenumber $k_{x}$ spectrum calculated directly from seismic data, as in Fig. 5. In comparison to Fig. 5, here the internal wave subrange is more prominent, while the turbulence subrange has a slope closer to $k_{x}{ }^{1}$ above wavenumbers of $\sim 0.02 \mathrm{cpm}$. The S/N ratio of this section of data is 6.2. (c) Seismic image showing tracked reflections (black lines) from the same section shown in (a), for a Fourier transform length of 256 points. White lines show reflector tracks for a Fourier transform length of 64 points. (d) Slope spectrum calculated from tracked reflections using a 256-point Fourier transform. Vertical lines show $95 \%$ confidence interval. Blue line shows turbulence fit, yellow line is internal wave subrange fit, and green line is example noise slope. Dashed lines show slope spectra for Fourier transforms of length 128 points (red), 64 points (cyan), and 32 points (green). Note the similarity of the reflector-based slope spectrum to the $k_{x}$ spectrum calculated from the $2 \mathrm{D}$ Fourier transform of the data (b).

carry little information about internal waves. These characteristics are expected, given that seismic wavelength is inversely proportional to frequency. Consequently, in using seismic data to estimate turbulence, it is important to use the highest possible frequencies that still have sufficient signal but not such high frequencies that random noise dominates.

Before selecting frequency ranges for filtering of seismic images prior to calculating $k_{x}$ slope spectra, passband analysis similar to that done in Fig. 9 should be done to determine which frequencies will be useful for that dataset. For line SO-1, frequencies above $80 \mathrm{~Hz}$ become noise dominated at high wavenumbers in slope spectra (curves 85, 95, and 105 in Fig. 9). The
$\mathrm{S} / \mathrm{N}$ ratios of each frequency band (Table 1 ) show that passbands with $\mathrm{S} / \mathrm{N}$ values below about 4 will be too noise dominated to produce reliable values in the turbulence subrange. An example of noise-dominated data from South China Sea line 5 (Fig. 10) has an $\mathrm{S} / \mathrm{N}$ ratio of 4.3 , and the slope spectrum shows a clear $k_{x}^{2}$ slope over wavenumbers above $0.006 \mathrm{cpm}$. For the SO-1 line, the analysis in Fig. 9 suggests that a frequency range of $30-80 \mathrm{~Hz}$ provides both a high $\mathrm{S} / \mathrm{N}$ ratio ( 8.3 for the entire section) and good sensitivity in the turbulence subrange. Lower frequencies have slightly higher $\mathrm{S} / \mathrm{N}$ ratios, but they lack sensitivity at the higher wavenumbers necessary to characterize the turbulence subrange. 


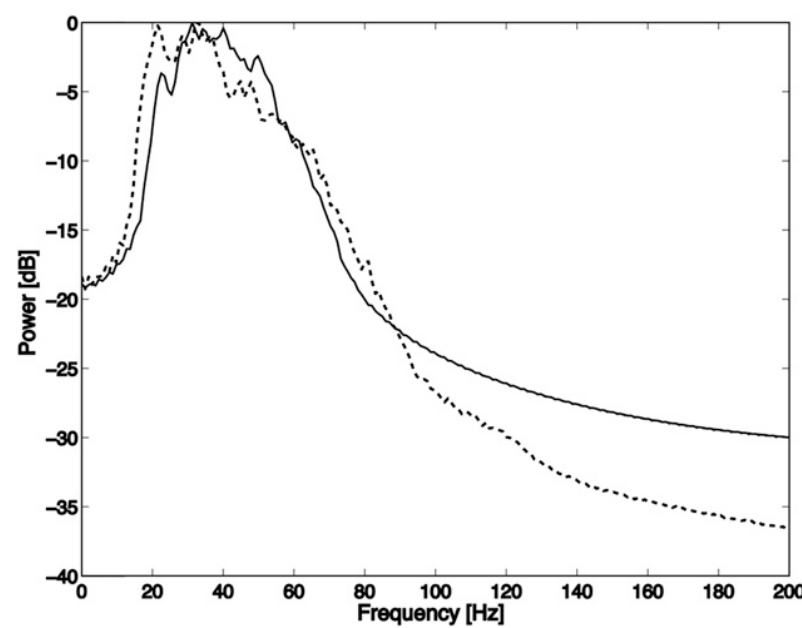

FIG. 7. Frequency spectrum of data on line SO-1 (dashed line; data section shown in Fig. 8) and South China Sea line 5 (solid line; data section shown in Fig. 5). Peak seismic frequency is approximately $30-40 \mathrm{~Hz}$; useful signal is limited to $<100 \mathrm{~Hz}$.

It should be noted that the spectral levels of $k_{x}$ slope spectra calculated directly from seismic images are strongly dependent on the frequency band of the seismic images. The dependence on frequency is much less when spectra are calculated from tracked reflectors, as will be seen below. Nevertheless, it is critical to conduct careful frequency and $\mathrm{S} / \mathrm{N}$ analysis of the data prior to tracking reflectors, to ensure that spectra are not unduly contaminated by noise.
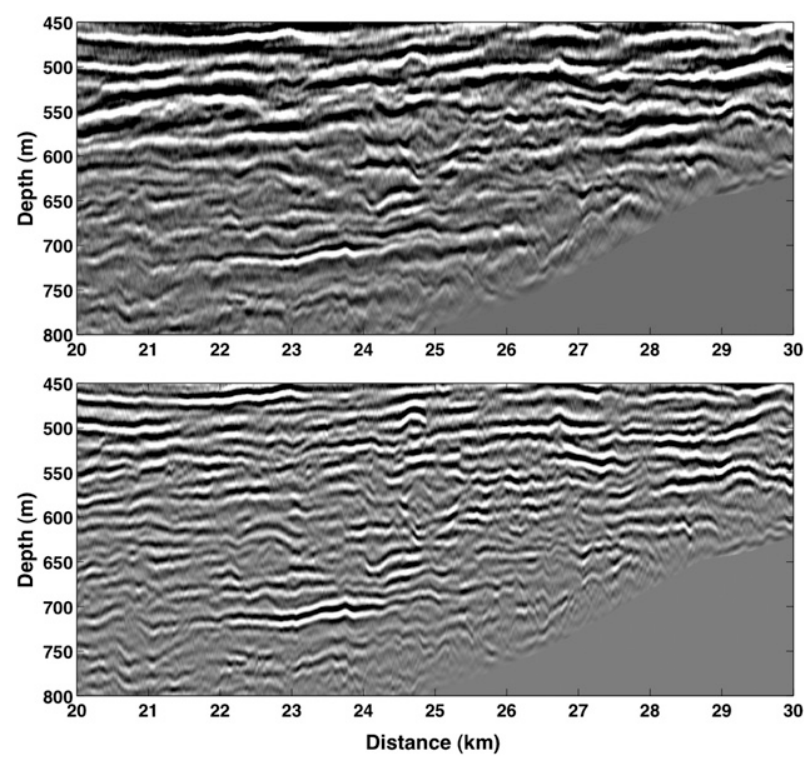

FIG. 8. Section of data from line SO-1, showing (top) unfiltered migration and (bottom) migration filtered from 40 to $80 \mathrm{~Hz}$. Note the enhanced detail in (bottom) due to filtering out of low frequencies.

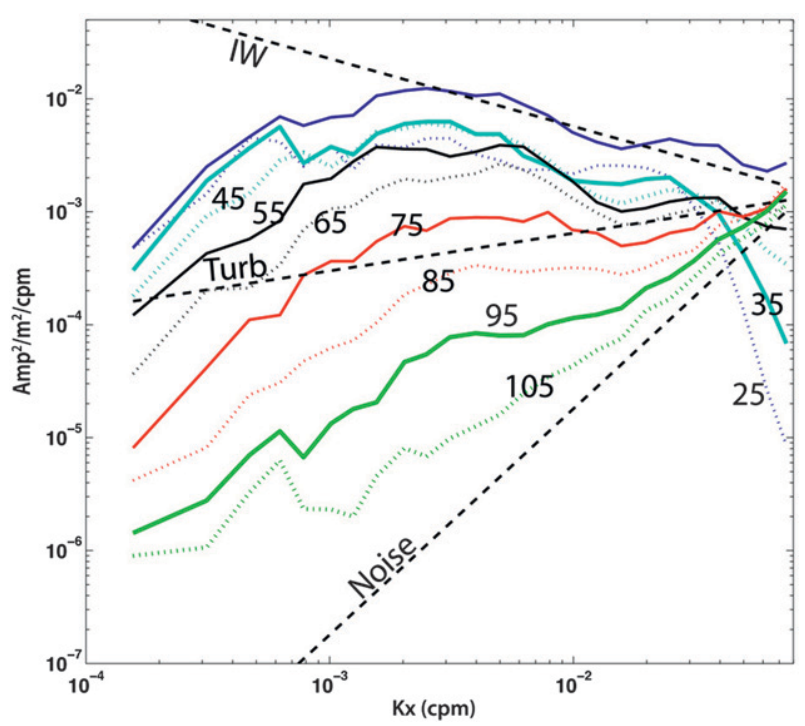

FIG. 9. Horizontal wavenumber $\left(k_{x}\right)$ slope spectra of different frequency passbands for offshore Nicaragua line SO-1. Passbands are $10 \mathrm{~Hz}$ in width, and the center frequency of each passband is labeled (e.g., $25=20-30 \mathrm{~Hz}$ passband, etc.). Solid blue line represents the preferred passband of $30-80 \mathrm{~Hz}$.

\section{e. Suppression of harmonic shot-generated noise}

An important, but generally overlooked, source of nonrandom noise in seismic data comes from the geometry of the seismic survey. This noise, which is especially significant when viewing seismic data in the horizontal wavenumber domain, exists at discrete wavenumbers related to the shot spacing at which the seismic data were acquired in the field. (Shot spacing is the horizontal distance between successive firings of the sound source, typically an array of air guns.) Specifically, the shot spacing creates harmonic noise at integer multiples of the shot spacing wavenumber. Denser shot spacing produces data with higher "fold," which is the number of seismic traces that image the same common midpoint (CMP) and are summed to make the final stacked trace. Shot spacing $\Delta_{s}$, the number $N$ of channels in the seismic

TABLE 1. S/N ratios of seismic data in passbands shown in Fig. 9.

\begin{tabular}{cc}
\hline \hline Frequency band $(\mathrm{Hz})$ & $\mathrm{S} / \mathrm{N}$ \\
\hline $20-30$ & 7.7 \\
$30-40$ & 7.8 \\
$40-50$ & 7.0 \\
$50-60$ & 6.1 \\
$60-70$ & 5.3 \\
$70-80$ & 4.1 \\
$80-90$ & 2.9 \\
$90-100$ & 1.7 \\
$100-110$ & 1.2 \\
$30-80$ & 8.3 \\
\hline
\end{tabular}



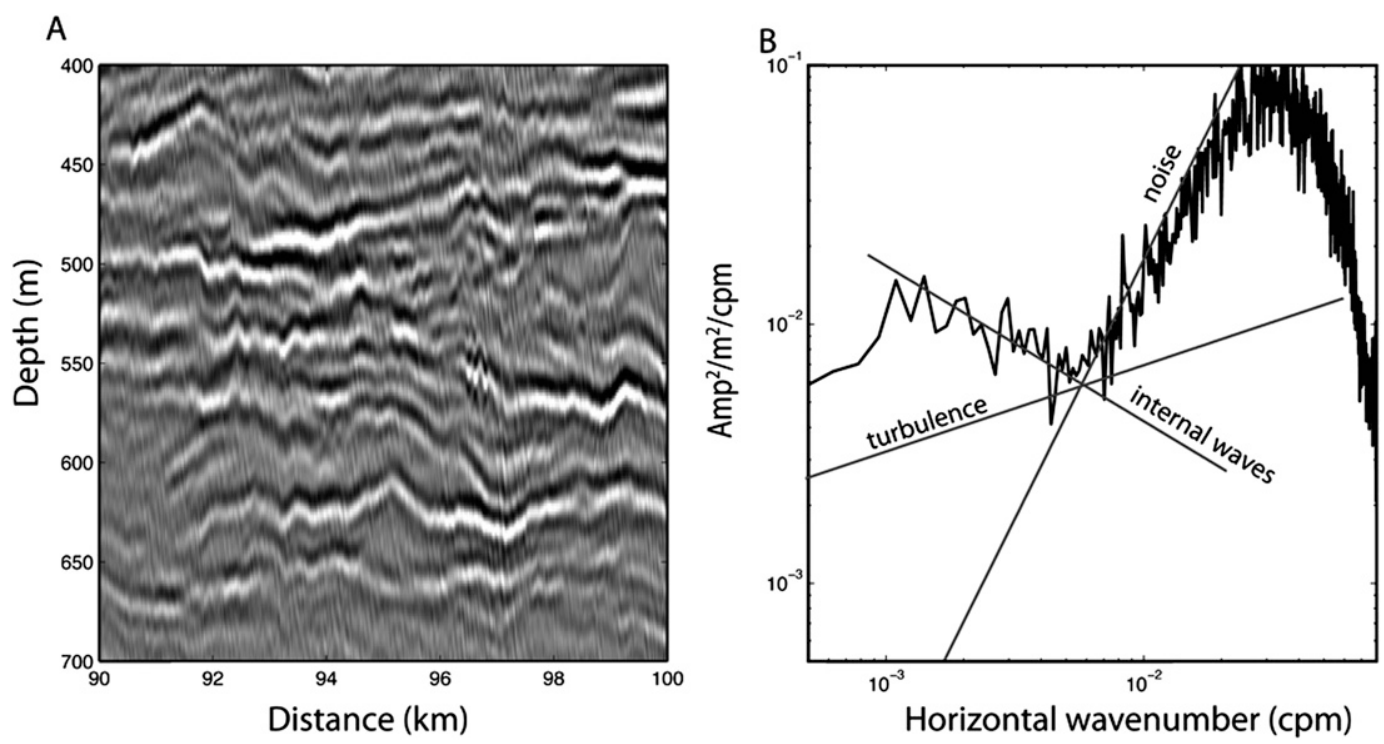

FIG. 10. (a) Noise-dominated data from South China Sea line 5 (data section from $x=90-100 \mathrm{~km}$ ), filtered from 30 to $60 \mathrm{~Hz}$. (b) Data transform of section shown in (a), in $k_{x}$ domain (bold black line). Gray lines show required slopes of turbulence, internal waves, and noise according to KM07 model. Note clear $k_{x}^{2}$ slope at wavenumbers above $\sim 0.006 \mathrm{cpm}$, indicating dominance of noise at these wavenumbers. $\mathrm{S} / \mathrm{N}$ ratio of this section is 4.4 , substantially lower than in the data sections of Figs. 5 and 6.

streamer, and the receiver spacing $\Delta_{x}$ define the seismic fold $F$ as $=N \Delta_{x} /\left(2 \Delta_{s}\right)$. Here, we discuss the cause, consequence, and suppression of harmonic $k_{x}$ noise on seismic slope spectra using two examples from the South China Sea: the relatively high-fold line 5, which has a shot spacing of $50 \mathrm{~m}$, and the lower-fold line 7, which has a shot spacing of $150 \mathrm{~m}$.

Along horizontal reflections, slight changes in the amplitude or arrival time of reflected energy on adjacent CMPs (stacked traces) create harmonic wavenumber $k_{x}$ noise. These amplitude fluctuations are entirely an artifact of survey shot spacing. The choice of shot spacing in a seismic survey is a compromise between the desire for high fold, which requires short shot spacing, and the desire to minimize reverberations, which requires long shot spacing. (The minimum boat speed required to keep the streamer trim, typically $\sim 5 \mathrm{kt}$, also places a practical constraint on the minimum shot spacing that can be achieved.) Spatial sampling for seismic reflection surveys is determined by the receiver spacing, as CMP spacing $\Delta_{\mathrm{CMP}}$ is half the receiver spacing. The number of CMPs between each shot $\left.N_{\mathrm{CMP}}\right)$ is $N_{\mathrm{CMP}}=\Delta_{S} / \Delta_{\mathrm{CMP}}$. Because $\Delta_{\mathrm{CMP}}$ is $6.25 \mathrm{~m}$ for data presented here, in the case of line 5, eight CMPs are produced between shots, so that the near-offset traces in every eight adjacent CMP gathers are from different receiver numbers on the streamer (the eight nearest to the source). This causes a periodic change in seismic amplitudes, which repeats every eight traces.
The harmonic shot spacing noise can be distinguished from signal in the frequency-wavenumber $(f-k)$ domain by passing data $g(t, x)$ through a two-dimensional fast Fourier transform (FFT):

$$
\begin{aligned}
& G(f, k)=F_{x}\left\{F_{t}[g(t, x)]\right\}, \\
& G(f, k)=F_{x}[G(f, x)],
\end{aligned}
$$

where $F_{t}$ and $F_{x}$ are the FFTs across time $t$ and distance $x ; G(f, k)$ is the complete 2D FFT. CMPs with identical near-offset receiver number and ray geometry occur at the shot spacing $\eta$. Noise spikes $k_{s}$ affect all frequencies with amplitudes proportional to $G(f)$ as an effect of $g(t, x)$ bandpass filtering, but occur only at regular wavenumber harmonics.

The primary cause of harmonic noise is the decay of amplitude $\Delta A_{\mathrm{CMP}}$ with longer raypaths and changing reflection geometries, which produces a periodicity of $\eta$ in the amplitude of the near traces on adjacent CMP gathers:

$$
\begin{aligned}
F_{t}\left\{F_{x}[g(t, x) s(x)]\right\} & =F_{t}\left[G(k) S_{k}\right], \\
G(f, k) & =G(f)\left[\sum_{n=0}^{\infty} a_{n} G\left(k-n k_{0}\right)\right],
\end{aligned}
$$

where $s(x)$ is a sawtooth function that describes the decay in amplitude and $\alpha_{n}$ is the coefficient for the Fourier 
A

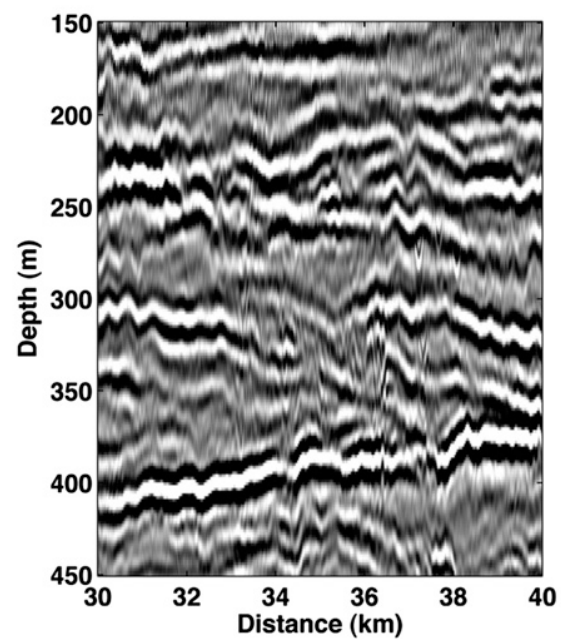

B

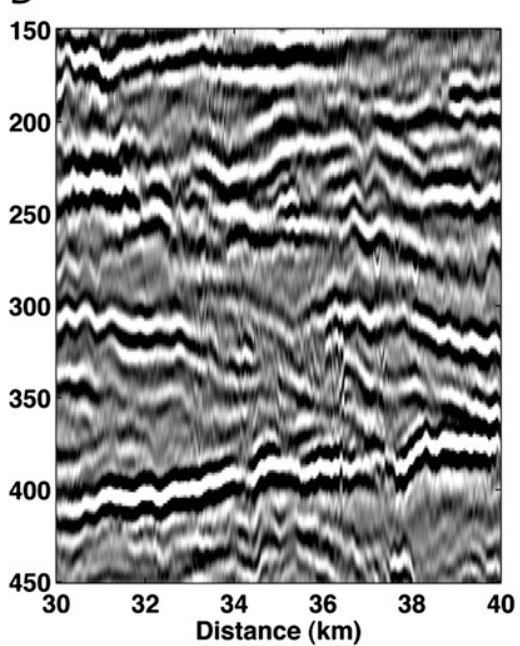

C

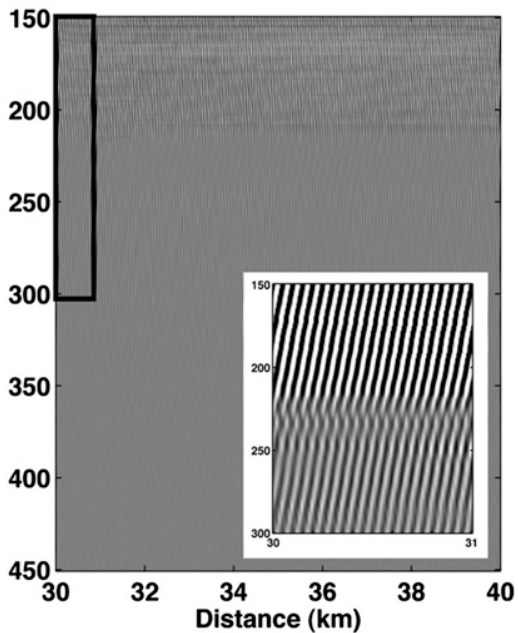

FIG. 11. Effect of $k_{x}$ filtering on seismic data, showing improved clarity and lateral continuity of shallow seismic reflections after removal of harmonic noise using the filter shown in Fig. 12. (a) Portion of original seismic section from South China Sea line 5. (b) Section after $k_{x}$ filtering. (c) Harmonic noise removed by the filtering, calculated as the difference between sections (a) and (b). Inset in (c) shows a zoom of the harmonic noise (black box); here, the fundamental mode of the noise is visible, with lateral spacing of $50 \mathrm{~m}$.

expansion of $a_{n}[s(x)]$. Additional harmonic noise may be present if normal moveout (NMO) corrections are not comprehensive, or if, as in the case of the direct arrival, there are other travel time effects on adjacent CMPs.

This harmonic noise is easily visible at shallow depth on seismic images as high-angle "cross hatching" (Fig. 11). Though present at all depths, the noise weakens with depth, as raypaths for adjacent CMPs become more similar. The harmonic impulses follow from the fact that the series expansions of $\alpha_{n}$ is periodic; this results in a line spectrum with harmonics at $k_{s}=n / \eta$, where $n$ is any integer, and $G(f)$ occur at several wavenumbers and are additive; a larger $\eta$ results in a more severely contaminated dataset. For the two examples used in this paper, with shot spacings of 50 and $150 \mathrm{~m}$, the first occurrence of $G(f)$ is at 0.02 (Fig. 12) and $0.00667 \mathrm{cpm}$ (Fig. 13), respectively, with further noise spikes visible at the integer harmonics (Figs. 12 and 13).

To remove these harmonics, we apply a $k_{x}$ domain filter designed to suppress the noise spikes, as follows. First, the data are transformed into the 2D Fourier domain as described above. When the sum of the absolute value of all frequencies for individual wavenumbers is plotted, $G(f)$ becomes apparent (Figs. 12 and 13). Noise spikes at $k_{x}=k_{s}$ are reduced by domain passing a bandstop notch filter centered over the spike in the wavenumber domain over all frequencies (Fig. 12,

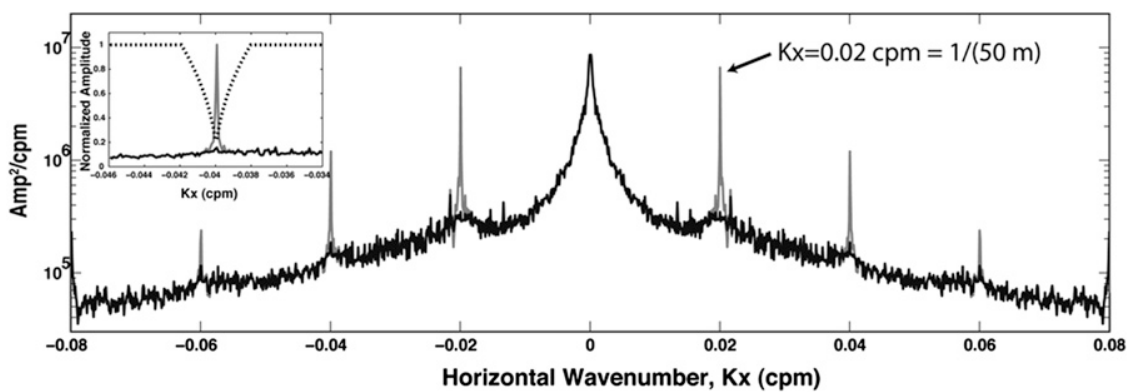

FIG. 12. Horizontal wavenumber $k_{x}$ spectrum of seismic data shown in Fig. 11, produced by summing the $2 \mathrm{D}$ Fourier $\left(f-k_{x}\right)$ spectrum along the $f$ axis. Gray and black lines show data before and after application of the $k_{x}$ notch filter, respectively. Prominent peak at $k_{x}=0.02 \mathrm{cpm}$ is the expression of amplitude changes in the CMP domain due to the shot spacing $[0.02 \mathrm{cpm}=$ $1 /(50 \mathrm{~m})]$; second noise peak at $\pm 0.04 \mathrm{cpm}$ is the first harmonic. Inset shows the shape of the $k_{x}$ filter (black dashed line) applied to suppress noise peaks. 


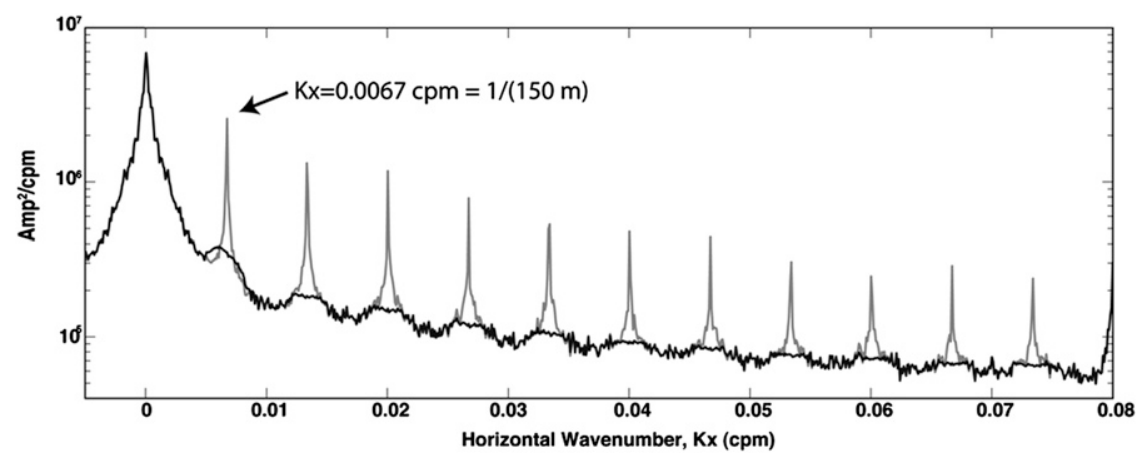

FIG. 13. The $k_{x}$ spectrum of seismic data from South China Sea line 7, plotted as in Fig. 12, but only positive $k_{x}$ are shown. These data were acquired with a coarse shot spacing of $150 \mathrm{~m}$. This produces a primary noise spike at $k_{x}=[1 /(150 \mathrm{~m})]=0.0067 \mathrm{cpm}$, with harmonic noise spikes at integer multiples thereof. Three iterations of the filter were applied to produce the bold line.

inset). The filtering process first isolates a spike and a variable amount of signal about the spike corresponding to the dimensions of the filter. The amplitudes of the isolated wavenumbers are detrended. Filtering is then done in the Fourier domain by multiplying the detrended amplitudes by the filter, which itself has a broad passband on either side of exponential transition bands (Fig. 12). The 2D inverse FFT is then calculated to recover the filtered seismic data (Fig. 11). The difference between filtered and unfiltered seismic data highlights the removed noise (Fig. 11). Unlike standard $f-k$ filters, this technique effectively removes the harmonic component from the high-fold dataset without eliminating high wavenumber data.

On the lower-fold dataset from line 7, the harmonic noise spikes are more closely spaced and begin at lower wavenumbers (Fig. 13). Because of the severity of the harmonic noise spikes, several successive applications of the filter are required to suppress the noise spikes (we used three iterations here). On these data, the filter is clearly less effective: spikes have been suppressed but remnants of the spikes are present near $k_{x}=k_{s}$ (Fig. 13). Moreover, the close spacing of the spikes raises questions about whether the underlying signal can be isolated. Clearly, caution must be used before interpreting slope spectra on seismic datasets acquired with coarse shot spacing. We discuss this further below.

To assess the effect of the $k_{x}$ filter on slope spectra, we compare the $k_{x}$ slope spectra of seismic data before and after filter application. Figure 14 shows this comparison for a portion of South China Sea line 7, before and after three iterations of the filter. The filter effectively removes the spikes associated with the harmonic noise (gray line, Fig. 14) and leaves the underlying signal
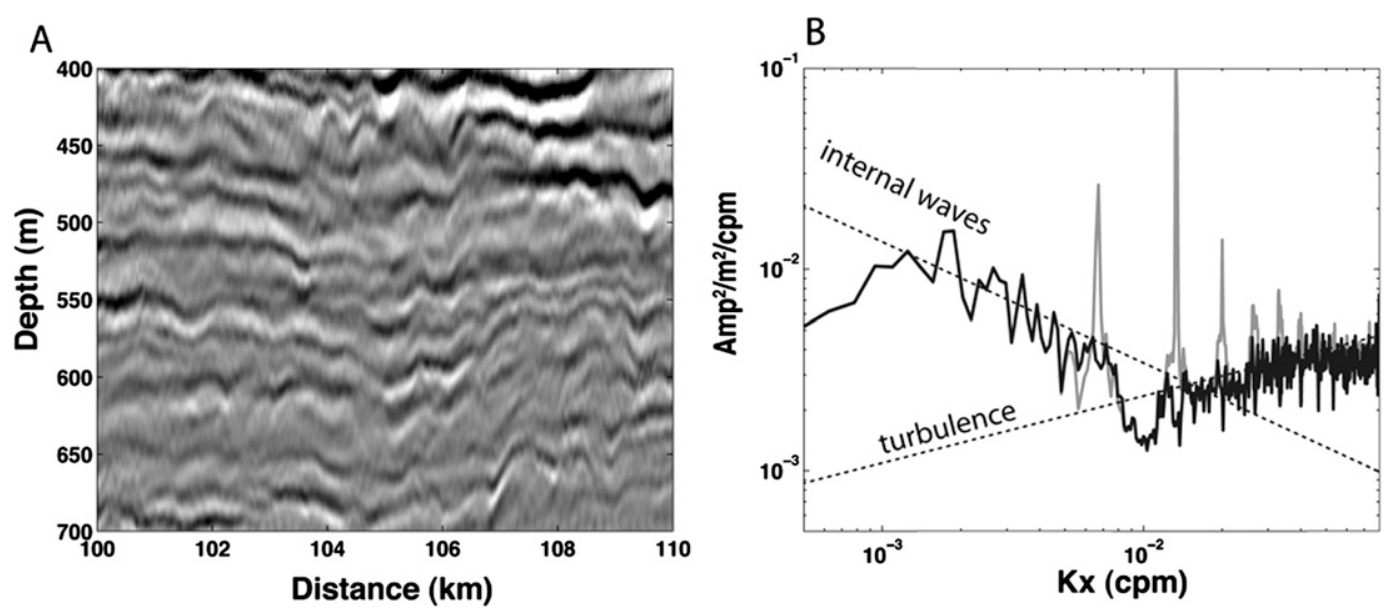

FIG. 14. (a) Portion of low-fold seismic data from South China Sea line 7 after $k_{x}$ filtering. (b) Slope spectrum calculated directly from seismic data before (gray) and after (black) three iterations of $k_{x}$ filter. Harmonic noise spikes are effectively removed by the filter. Dashed lines show required slopes of internal wave and turbulence subranges from the KM07 model. 
unchanged. The internal wave subrange is unaffected by the filter; above wavenumbers of $0.01 \mathrm{cpm}$ (wavelengths less than $100 \mathrm{~m}$ ), the filtered slope spectrum has a slope very close to the expected turbulence subrange of $\left(k_{\mathrm{x}}\right)^{1 / 3}$. These results suggest that $k_{\mathrm{x}}$ filtered seismic data can be used to extract quantitative slope spectral estimates from the internal wave subrange, and possibly from the turbulence subrange. However, residual harmonic noise in the turbulence subrange suggests that caution should be used in interpreting turbulence values from low-fold seismic data.

\section{f. Reflector slope spectra}

Horizontal wavenumber spectra calculated from the seismic data (e.g., Fig. 5b) accurately represent the horizontal wavenumber content inherent in the seismic images, but they cannot provide turbulence estimates directly, as they cannot be readily scaled to represent displacement spectra, for several reasons. First, the seismic data values recorded in the $x-t$ domain are seismic amplitudes, not meters of vertical displacement. Therefore, to achieve displacement spectra in the horizontal wavenumber domain, we must first extract vertical displacement profiles from the seismic images, which we accomplish by tracking reflections (as described below). Second, the $k_{x}$ spectra derived directly from seismic data will be influenced by seismic amplitudes, for reasons that have nothing to do with vertical displacements. Reflection strength in a seismic section is primarily a function of the size of the temperature "step" responsible for generating the reflection (modulated by convolution with the source signal). Upon Fourier transform, strong reflections will thus have undue influence in the spectral domain. Finally, regions dominated by noise will affect data transforms but are readily excluded by tracking algorithms, which rely on similarity of adjacent seismic traces to accomplish the tracking.

A key assumption in using tracked reflections to extract $k_{x}$ spectra is that the reflectors follow isopycnalsthat is, that displacements in reflectors represent isopycnal displacements. Permanent fine structure due to features such as intrusions or thermohaline staircases will appear as seismic reflections. While these features have slight slopes with respect to the density surfaces (see, e.g., Ruddick 1992), the slope spectra will be dominated by the wave and turbulence part in the wavenumber range studied here. When fine structure is reversible, for example, caused by internal wave strains, the displacements in reflectors may not be indicative of isopycnal displacements. Of critical importance is the time scale over which fine structure persists, in comparison to the time scale over which displacements are imparted by the ambient internal wave and turbulence fields. If fine

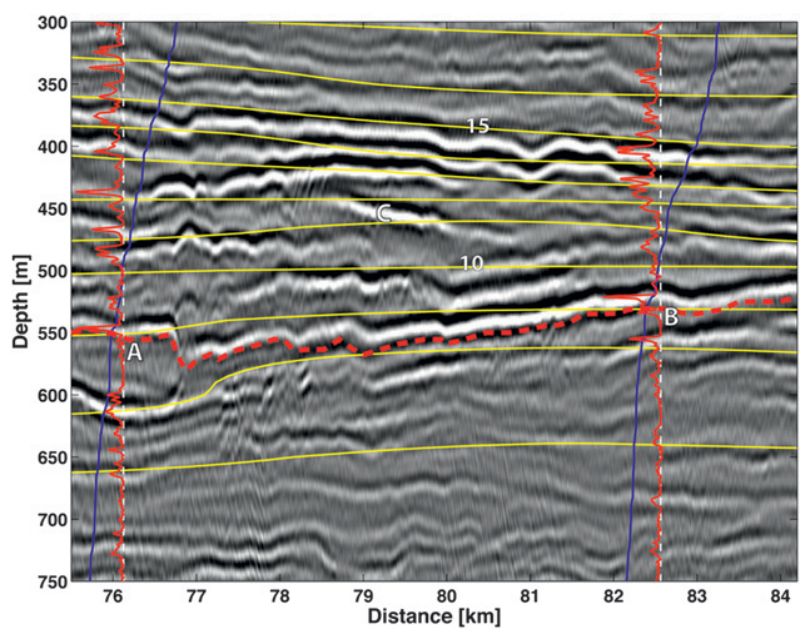

FIG. 15. Portion of South China Sea line 5 seismic section, showing locations of XBT drops (white dashed lines), temperature profiles (blue lines), isotherms (green lines, labeled in ${ }^{\circ} \mathrm{C}$ ), and vertical temperature gradients (red solid lines). Dashed red line and points $\mathrm{A}, \mathrm{B}$, and $\mathrm{C}$ are discussed in the text. Reflections generally follow isotherms closely. Note the close correspondence between seismic reflections and areas of strong vertical temperature gradient $d T / d z$. Isotherms were calculated using temperature-depth data from all XBTs on the line (Fig. 1), using the "blockmean" and "triangulate" functions in Generic Mapping Tools (Wessel and Smith 1991), which perform block averaging and optimal Delauney triangulation (Watson 1982).

structure persists over sufficiently long time scales, then even reversible fine structure will record the ambient displacement fields.

We can assess whether reflections follow isotherms in the data used here, since both surveys included coincident temperature data from expendable instruments deployed during seismic shooting. Reflection images from South China Sea line 5 and Nicaragua line SO-1, together with temperature data and isotherms calculated from XBT drops, are shown in Figs. 15 and 16, respectively. In both surveys, temperature profiles show little evidence for intrusions, as temperature inversions are rare; rather, temperature fine structure largely consists of high-gradient "steps" separating layers of lower vertical temperature gradient. Nevertheless, both datasets show reflections that, with few exceptions, follow isotherms. In the South China Sea section, several strong reflections follow isotherms over lateral distances up to $10 \mathrm{~km}$ (e.g., the reflection following the $15^{\circ} \mathrm{C}$ isotherm in Fig. 15). A few reflections may cross isotherms, such as the short reflection labeled " $C$ " and the reflection immediately above it. However, even some instances where, at first glance, reflections appear not to follow isotherms may be artifacts of the XBT spacing and temperature contouring. For example, the reflection connecting points " $A$ " and "B" in Fig. 15 appears to 


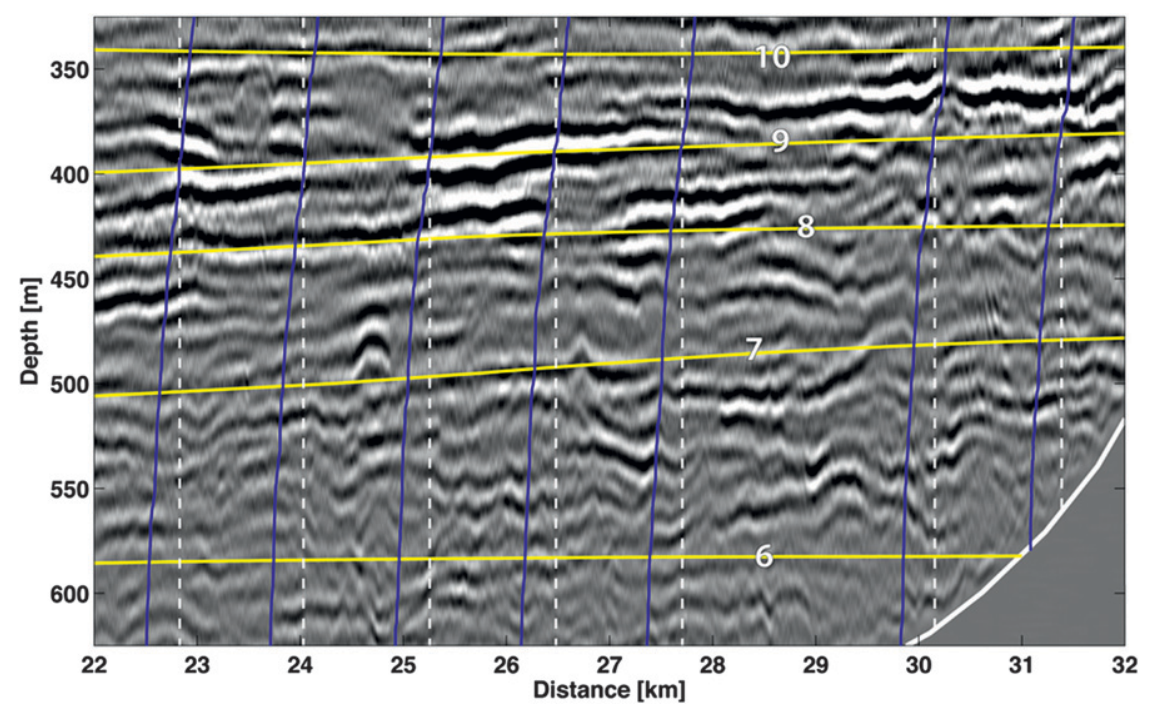

FIG. 16. Portion of seismic section from offshore Nicaragua line SO-1, showing locations of XBT drops (white dashed lines), in situ temperature profiles (blue lines), and isotherms (green lines, labeled in ${ }^{\circ} \mathrm{C}$ ). Solid white line indicates seafloor. Most reflections follow isotherms over horizontal scales of $\sim 1 \mathrm{~km}$.

cross the $9^{\circ} \mathrm{C}$ isotherm in the middle of the section shown. However, since no temperature data exist between the two nearest XBTs, the temperature contours are necessarily smoother than the true isotherms. The dashed red line in Fig. 15 shows a reasonable interpretation of the $9^{\circ} \mathrm{C}$ isotherm, hand drawn so as to intersect the $9^{\circ} \mathrm{C}$ value at the XBTs while still following the entire reflection. This is a universal situation in comparing in situ temperature data to seismic sections: since the seismic images have lateral resolution far superior to that of even the densest XBT surveys, reflections show short-wavelength fluctuations that cannot be captured by the XBT surveys. In general, in the South China Sea line analyzed here, reflections principally appear to follow isotherms.

The offshore Nicaragua dataset has more densely spaced XBTs, so the relationship between reflectors and isotherms is less ambiguous. On this section, reflections appear generally parallel to isotherms at lateral scales of $\sim 1 \mathrm{~km}$ (Fig. 16). At longer length scales, however, some fine structure may be slightly inclined with respect to isotherms. The strong reflection following the $9^{\circ} \mathrm{C}$ isotherm at $\sim 27.5 \mathrm{~km}$, for example, appears to be slightly more strongly inclined downward to the left of the section. Over a lateral distance of $\sim 5 \mathrm{~km}$, the reflection departs from the $9^{\circ} \mathrm{C}$ isotherm by about $15 \mathrm{~m}$. However, in calculating slope spectra, we generally use reflectors of length 128 or 256 traces, that is, with a maximum length of $1.6 \mathrm{~km}$. Over these lateral scales, with rare exceptions, we see little evidence that reflectors depart significantly from isotherms in our datasets.
Before producing slope spectra, we need to track reflections in the seismic data. Various approaches to reflector tracking have been used, including user-guided amplitude tracking (Holbrook and Fer 2005) and crosscorrelation methods (Krahmann et al. 2008). We tracked reflections in the seismic data using an approach that relies on the instantaneous phase angle $\phi$ from the Hilbert transform (e.g., Yilmaz 1987), a seismic attribute that highlights reflection continuity (Barnes 2007). We contoured the 0.6 value of $\cos (\phi)$, which is a smooth function that eliminates the $\pi / 2$ phase angle discontinuities present in $\phi$ itself. The contours were then divided into continuous reflector segments of minimum length $n$ data points, where $n=32,64,128$, or 256 . Tests on synthetic data show that this approach produces accurate reflector tracks that are more reliable than tracking reflection amplitudes and less time consuming to produce than user-guided picks. An example of the reflectors' tracks produced by this method is shown in Fig. 5c.

We produce "reflector slope spectra" from tracked seismic reflections as follows. Tracked reflections are divided into half-overlapping segments of length $n$ points, where $n$ is the length of the Fourier transform and is detrended prior to Fourier transformation. We calculated spectra for several choices of $n(32,64,128$, and 256), corresponding to reflector segment lengths of 200-1600 m. Following KM07, reflector slope spectra $\phi_{R x}$ are calculated as $\phi_{R x}=\left(2 \pi k_{x}\right)^{2} \phi_{R}$, where $\phi_{R}$ is the displacement power spectrum, calculated as in Holbrook and Fer (2005), and $R_{x}$ indicates that we are using reflector 
displacements rather than isopycnal displacements $\left(\zeta_{x}\right.$ in KM07). Before averaging, spectra for each reflector are scaled by the local buoyancy frequency at the reflector depth, $N(z)$, which is determined from the nearest XCTD probe in the offshore Nicaragua dataset, and from a regional profile in the South China Sea (L. St. Laurent 2010, personal communication). In the region of interest, turbulence dissipation is then estimated by fitting the mean reflector slope spectra to the KM07 model for the turbulence subrange [Eq. (12) of Klymak and Moum 2007b], using a nonlinear least squares inversion. Eddy diffusivity is then estimated as $K_{\rho}=0.2 \varepsilon N^{-2}$.

An example of a slope spectrum calculated from tracked reflectors in the South China Sea line 5 is shown in Fig. 5d. It is instructive to compare the "data slope spectrum" of Fig. 5b with the "reflector slope spectrum" of Fig. 5d. The two spectra show similar characteristics, with a discernible break between the internal wave subrange and the turbulence subrange, which has a clear slope of $k_{x}^{1 / 3}$. Both spectra show a drop-off at wavenumbers greater than $0.04 \mathrm{cpm}$ (wavelengths smaller than $25 \mathrm{~m}$ ). Differences between the two spectra are expected, since the reflector slope spectrum only incorporates a limited number of successfully tracked reflectors (black lines in Fig. 5c), whereas the data slope spectrum includes the entire image. Because the tracking algorithm sifts high-amplitude reflections and excludes transparent zones, the reflector spectrum is likely less susceptible to noise than the data spectrum.

A second example of a reflector slope spectrum, from South China Sea line 5 (Fig. 6), shows the effect of the selected Fourier transform length on interpreted turbulence levels. The reflector slope spectrum (Fig. 6d) and its corresponding data slope spectrum (Fig. 6b) both show a sharp transition between the internal wave subrange and the turbulence subrange at $k_{x}=0.025 \mathrm{cpm}$. Interestingly, the tracked reflector slope spectrum is inclined at exactly the expected value of $k_{x}^{1 / 3}$ in the turbulence subrange, even though the data slope spectrum showed a slightly higher value. This is likely a consequence of noisy patches in the data that do not affect the tracked reflectors, which preferentially follow reflections with a higher signal-to-noise ratio. Choosing a shorter Fourier transform length (e.g., 64 points) allows tracking of shorter reflections (white lines, Fig. 6c), which sample areas of lower signal-to-noise ratio than the longer tracks and produce higher estimates of turbulence (dashed lines, Fig. 6d). This dependence of turbulence estimates on Fourier transform length is an important point: shorter FFT lengths allow reflections to be tracked in areas of lower signal, which are expected to be areas of higher turbulence (signal-to-noise ratio, by definition, is a measure of the similarity of adjacent CMP-stacked seismic traces, which should be suppressed by turbulence).

\section{g. Estimation of diffusivity from XCPs}

Coincident with seismic data acquisition on line SO-1 off Nicaragua, we deployed 26 XBTs and three XCPs (Fig. 1). The XCP measures the weak electrical potentials generated by the flow of the conductive ocean in the magnetic field of the earth to derive profiles of the horizontal velocity (Sanford et al. 1982). Typical uncertainties in $2-\mathrm{m}$ binned velocities are $0.5 \mathrm{~cm} \mathrm{~s}^{-1}$ (Kunze et al. 2002).

Estimates of vertical diffusivity $K_{\rho}$ were derived from the XCP velocity and temperature profiles using the technique of Henyey et al. (1986), Gregg (1989), and Polzin et al. (1995). Salinity profiles were developed using the temperature-salinity relationship from the nearest CTD station obtained by the R/V New Horizon during the cruise. From these profiles we computed density and buoyancy frequency $N$ profiles for each $\mathrm{XCP}$. The Henyey-Gregg-Polzin technique uses the vertical shear in the horizontal velocities on 10-m scales $\left(S_{10}\right)$ in an empirical relation that estimates the turbulent dissipation rate $\varepsilon$ and is related to eddy diffusivity as $K_{\rho}=0.2 \varepsilon N^{-2}$ (Osborn 1980). The basic relationship, which depends on the fourth power of $S_{10}$ and the shearto-strain ratio $R_{\omega}$, has been validated by several field programs (Polzin et al. 1995) using combined velocity and turbulence profilers and applied several times to XCP data (D'Asaro and Morison 1992; Lee et al. 2006; Nash et al. 2007). The main problem with application of the scaling is in low-stratification regions (Kunze et al. 2006), which is not a concern for these relatively shallow casts. For these data, a running 100-m window of 10-m shear variance [obtained from 10-m linear fits to the raw data and multiplied by 2.11, following Gregg (1999)] and buoyancy frequency was used to estimate the diffusivity profiles. The latitudinal dependence of the scaling was included (Gregg et al. 2003). The shear-to-strain ratio was obtained as the ratio of vertical wavenumber spectra of $N$-normalized shear and strain, each integrated to $0.1 \mathrm{cpm}$. The spectra are calculated using 128-m-long segments. The strain spectra are obtained from $N^{2}$ profiles, approximating the background stratification using a quadratic fit, and correcting for the first differencing inherent in the gradients. Spectral analysis of the XCP data suggests a rather high shear-to-strain ratio of $R_{\omega}=17$. When averaged using the data points where $N>4 \times$ $10^{-3} \mathrm{~s}^{-1}$, that is, excluding relatively weak stratification that can erroneously increase the normalized-shear variance, $R_{\omega}=8$. We used $R_{\omega}=17$ in our application to the seismic data (section 4b). Calculations using $R_{\omega}=8$ 
A
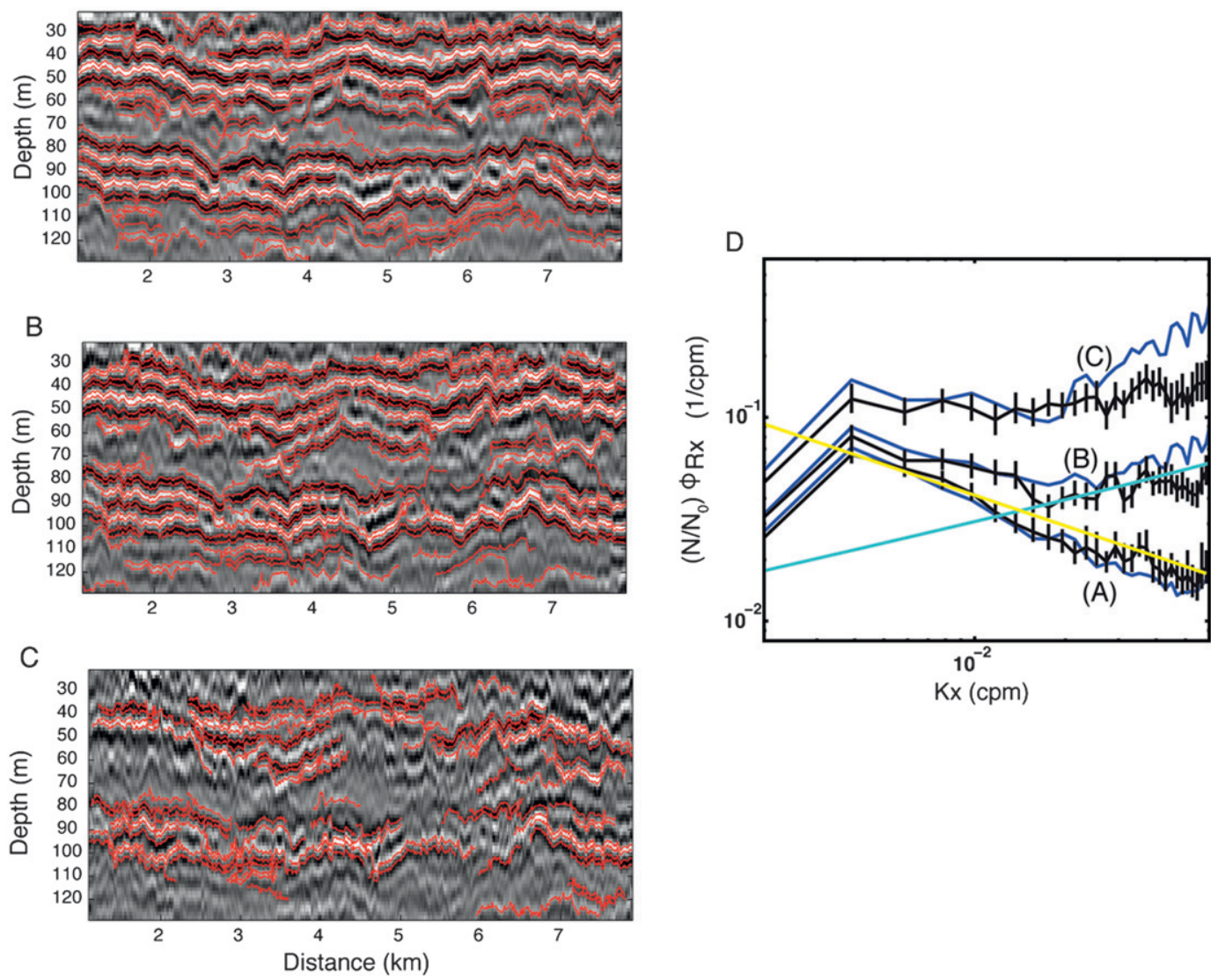

FIG. 17. Synthetic seismic sections calculated from the sound speed model of Fig. 3, for three levels of turbulence: (a) low, (b) moderate, and (c) high. Red lines show tracked reflections in each section. Note the increased choppiness of reflections (and hence fine structure) as turbulence increases. (d) Slope spectra calculated from tracked reflections for each synthetic section (black lines with $95 \%$ confidence intervals) plotted with slope spectra calculated from isopycnals from each synthetic model (blue lines). Spectra were calculated for 64-point reflector lengths (corresponding to $512 \mathrm{~m}$ for the synthetic dataset). Yellow and blue lines show required slopes of internal wave (yellow) and turbulence (blue) subranges.

would require a correction larger by a factor of 1.6. The uncertainty in our estimates of $K_{\rho}$ using XCPs should be comparable to or better than that inferred for shear measurements using lowered acoustic Doppler current profilers, which require significant spectral corrections (Polzin et al. 2002). While it would have been desirable to have obtained coincident dissipation measurements from a free profiler, this is not possible from a constantly moving seismic ship, and the XCP offered the best method of estimating the dissipation rate of turbulent mixing.

\section{Results}

\section{a. Synthetic tests}

Synthetics were calculated for three increasing levels of turbulence (Fig. 17). The turbulence levels were selected arbitrarily, but, assuming a constant buoyancy frequency of $2 \mathrm{cph}$, they produce isopycnal displacements that approximate those expected for vertical diffusivity levels of $2 \times 10^{-6}, 2 \times 10^{-5}$, and $1 \times 10^{-4} \mathrm{~m}^{2} \mathrm{~s}^{-1}$. The appearance of the seismic image changes markedly as turbulence increases, from long, relatively continuous reflections at low turbulence to increasingly choppy and discontinuous reflections at high turbulence (Fig. 17).

Reflector tracks tend to follow high-amplitude events in the image, with relatively few tracks in areas of low signal, where the vertical temperature (and sound speed) gradients are lower. Tracking reflections becomes more difficult as turbulence increases because of the increased choppiness of reflections. In these synthetic examples, this does not bias the results, since the synthetic models have equal levels of turbulence everywhere. In real data, however, where spatially variable turbulence is expected, 


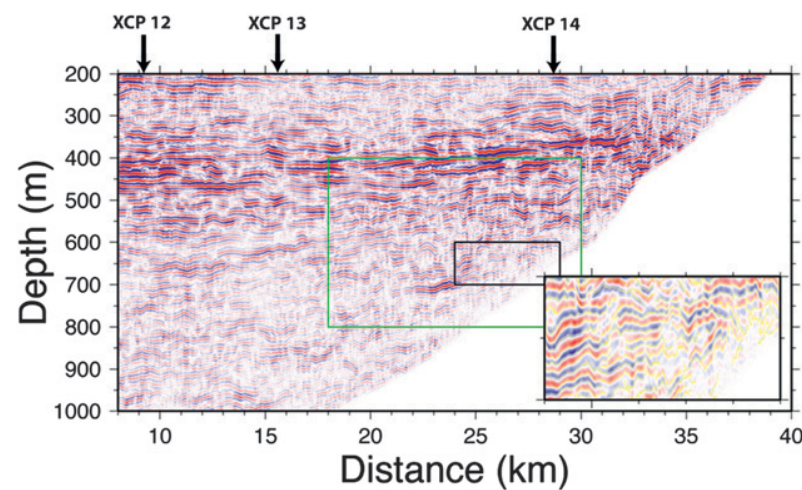

FIG. 18. Portion of seismic line SO-1 used for turbulence analysis. Green box outlines portion of data used in Fig. 20. Inset shows zoom of data in small black box, showing tracked reflections using $n=32$ (yellow lines). Locations of XCPs are shown by arrows.

this will likely bias the results toward lower values of turbulence.

Overall the reflector slope spectra provide an acceptable match to the isopycnal slope spectra, with fidelity that decreases with increasing turbulence. Reflector slope spectra match at all wavenumbers for the low-turbulence example: out to $k_{x} \sim 0.04 \mathrm{cpm}$ for the medium-turbulence example and out to $k_{x} \sim 0.015 \mathrm{cpm}$ for the high-turbulence example. Since sound speed structure becomes more complex as turbulence increases, lateral propagation effects and migration artifacts may contribute to this loss of signal. We do not use wavenumbers higher than $0.04 \mathrm{cpm}$ to interpret turbulence. We note that, as turbulence increases, signal-tonoise levels decrease markedly in the synthetic seismic data, from 11.1 in the low-turbulence section to 5.4 for the high-turbulence example. This is expected, since signal-to-noise levels measure trace-to-trace coherency and increased turbulence renders adjacent seismic traces less similar. This demonstrates that higher turbulence leads to lower reflected signal; in the limit, where turbulence produces vertically homogeneous layers, no reflected signal will result, and seismic images will be transparent.

These results demonstrate that the reflector slope spectra can reproduce isopycnal slope spectra with reasonable fidelity, as long as fine structure is "permanent." More work is needed to investigate the $k_{x}$ signatures of fine structure generated in more realistic, dynamic ocean models. However, the synthetics show clearly that, for standard seismic data frequencies $(30-80 \mathrm{~Hz})$ and horizontal sampling $(6.25 \mathrm{~m})$, we should expect sensitivity to horizontal wavenumbers out to about $0.04 \mathrm{cpm}$, depending on the level of turbulence.

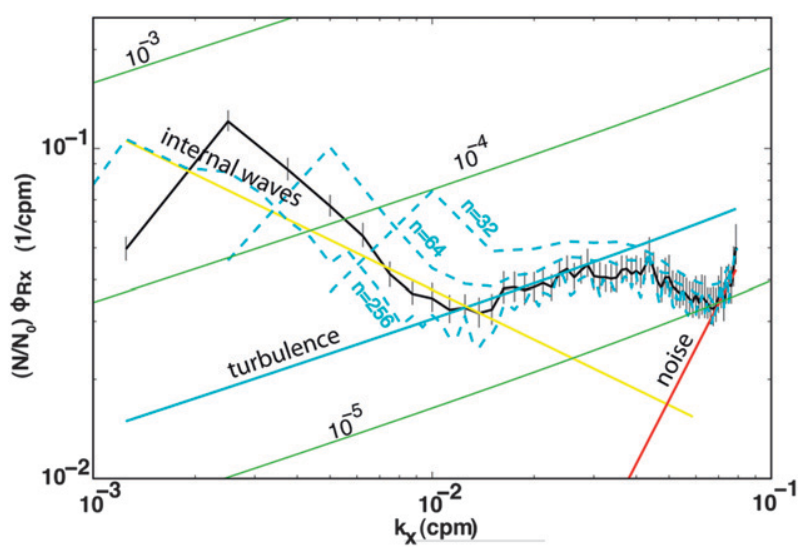

FIG. 19. Mean reflector slope spectra calculated for tracked reflections for $n=128$ (bold solid line), with 95\% confidence interval (vertical lines) shown for 161 degrees of freedom assuming vertical decorrelation over six reflectors; this is a conservative error estimate. Dotted lines show slope spectra calculated with other values of $n(32,64,256)$. Best-fitting lines for internal wave subrange (yellow line), turbulence subrange (cyan line), and noise (red line) are shown for the mean spectrum with $n=128$. Green lines show spectral levels for $K_{\rho}$ values of $10^{-3}, 10^{-4}$, and $10^{-5} \mathrm{~m}^{2} \mathrm{~s}^{-1}$.

\section{b. Turbulence dissipation from offshore Nicaragua seismic data}

We tracked reflections in line SO-1, offshore Nicaragua, as described above. We chose the Nicaragua dataset because coincident XCP data there enable a comparison of turbulence dissipation estimates from seismic data to in situ estimates (section 4c). Figure 18 shows an example of tracked reflections in that dataset.

The resulting reflector slope spectra show a remarkable fit to the expected shape of the turbulence subrange. Figure 19 shows mean reflector slope spectra for 425 individual reflectors in a 12 -km-wide $\times 400$-m-high area of the seismic section (green outline, Fig. 18). At $k_{x}>0.01 \mathrm{cpm}$ (horizontal wavelengths $<100 \mathrm{~m}$ ), the slope spectra show a distinct change to a $k_{x}{ }^{1 / 3}$ slope, which persists out to $k_{x} \sim 0.07 \mathrm{cpm}$. At the very highest wavenumbers, the data may show the $k_{x}^{2}$ spectral slope consistent with noise. For $k_{x}<0.01 \mathrm{cpm}$, the slope spectra show an acceptable fit to the internal wave subrange of KM07 (slope $k_{x}^{-1 / 2}$ ), especially for longer $n$, although our spectra appear to be somewhat steeper than KM07. A histogram of slope of the low-wavenumber portion of spectra inferred from tows of KM07 show a broad distribution between -2 and 1 (their Fig. 10); hence the deviations from the GM75 slope are expected. Turbulent wavenumbers $(0.01-0.04 \mathrm{cpm})$ of the observed slope spectrum are fit to the model spectrum [Eq. (2)] by varying the dissipation rate to minimize the residual using the Levenberg-Marquardt algorithm 

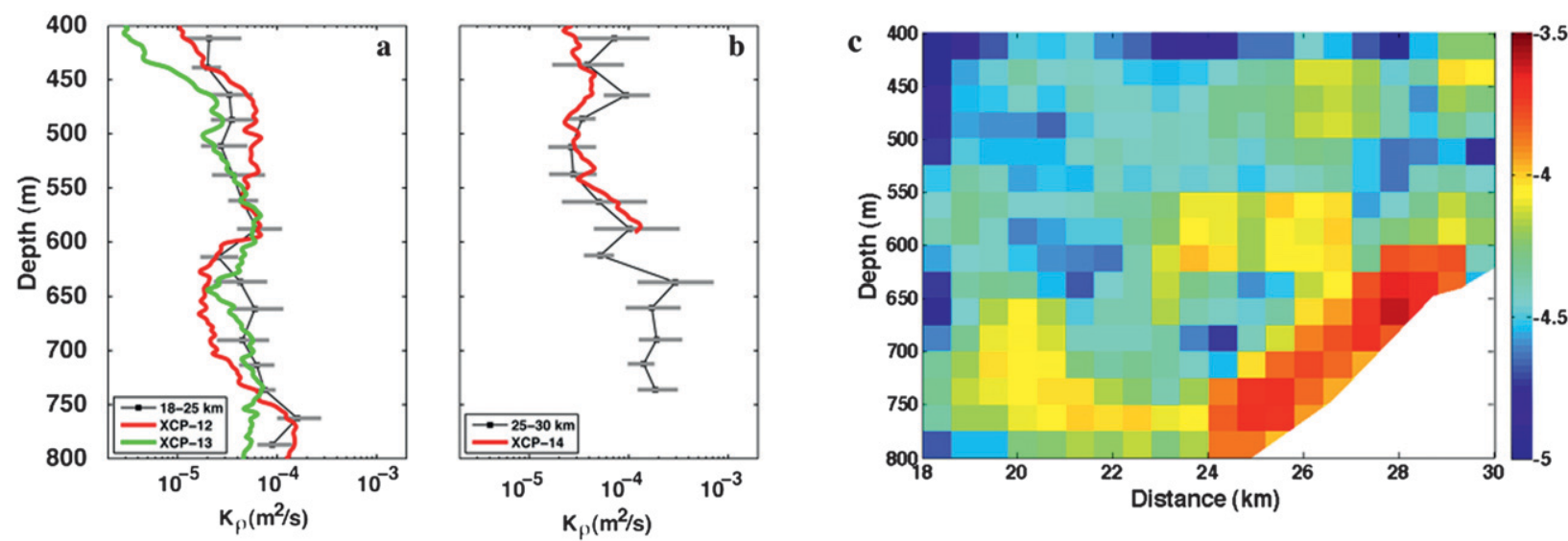

FIG. 20. (a) Depth profiles of mean $K_{\rho}$ estimates from reflector slope spectra in offshore portion (18-24 km) of seismic profile in Fig. 18 (squares) compared to $K_{\rho}$ values calculated from $10-\mathrm{m}$ shear on XCPs 12 and 13 (colored symbols; locations shown in Fig. 18). Profiles from reflector slope spectra are averaged in $25-\mathrm{m}$ vertical bins and are shown with $95 \%$ bootstrap confidence intervals inferred from 1000 bootstrap resamples of the data points in each depth bin. Uncertainty in estimates of $K_{\rho}$ from XCP data (not shown for clarity) are approximately a factor of 3 , similar to the size of error bars on seismically derived estimates. (b) As in (a), except comparing the nearshore portion of seismic section $(25-30 \mathrm{~km})$ and XCP 14. (c) Spatial distribution of $K_{\rho}$ estimates in green box of Fig. 18, averaged in boxes $600 \mathrm{~m}$ wide $\times 25 \mathrm{~m}$ high and then smoothed with a $1 \times 1$ boxcar function. Scale at right shows $\log _{10}\left(K_{\rho}\right)$.

for nonlinear least squares. The $95 \%$ confidence level is then obtained, for each spectrum, from the residuals. The confidence limits varied between a factor of 1.3 and 2.4 , with mean and standard deviation of $1.6 \pm 0.15$. The fit to the internal wave subrange is not removed from the observed spectrum, as the variability of the slope in the internal wave subrange might affect the quality of the results in the turbulence subrange. The value of $K_{\rho}$ that best fits the slope spectra for $n=128$ over the $k_{x}$ range from 0.01 to $0.04 \mathrm{cpm}$ is $2.3 \times 10^{-5} \mathrm{~m}^{2} \mathrm{~s}^{-1}$; $95 \%$ confidence intervals suggest acceptable fits between $\sim 1.6 \times 10^{-5}$ and $3.1 \times 10^{-5} \mathrm{~m}^{2} \mathrm{~s}^{-1}$. The $K_{\rho}$ estimates depend on the choice of $n$ (Fig. 19), with smaller values of $n$ producing higher $K_{\rho}$; this is not an error, but rather a consequence of the fact that the reflector tracks themselves depend on $n$ (e.g., Fig. 6c). A smaller $n$ allows shorter reflector segments to be tracked and included in the slope spectra; since shorter reflector segments are typically concentrated in "disrupted" areas such as the continental slope, the result is a higher (and likely more accurate) estimate of $K_{\rho}$ with greater spatial resolution. In our section, $K_{\rho}$ estimates increase from $1.6 \times 10^{-5} \mathrm{~m}^{2} \mathrm{~s}^{-1}$ for $n=256$ to $4.6 \times 10^{-5} \mathrm{~m}^{2} \mathrm{~s}^{-1}$ for $n=32$.

\section{c. Comparison to in situ data}

We tested our seismic-based $K_{\rho}$ estimates against in situ data by comparing to the $K_{\rho}$ values derived from coincident, concurrent XCP data (Figs. 20a and 20b). The seismic and $\mathrm{XCP}$ estimates show a remarkable match, both in terms of the average $K_{\rho}$ values $(4.4 \times$ $10^{-5} \mathrm{~m}^{2} \mathrm{~s}^{-1}$ for the XCPs, $4.6 \times 10^{-5} \mathrm{~m}^{2} \mathrm{~s}^{-1}$ for the seismic estimate) and the trends with depth. In Figs. 20a and 20b we separate near-slope XCP14 from the deeperwater XCPs 12 and 13 and compare them to the appropriate sections of the seismic line. The comparison shows a compelling match: the seismic-derived estimates match not only the overall levels of $K_{\rho}$ (between $10^{-5}$ and $10^{-4} \mathrm{~m}^{2} \mathrm{~s}^{-1}$ ) but also the downward increase in $K_{\rho}$ as well as several finer-scale features, such as the local peak in $K_{\rho}$ at depths of $\sim 600 \mathrm{~m}$. The match between in situ and seismic estimates of $K_{\rho}$ strongly supports the inference that reflector slope spectra can produce quantitative estimates of vertical diffusivity in the ocean.

Our results enable estimates of the spatial distribution of $K_{\rho}$ across the seismic section (Fig. 20c). This is done by calculating the diffusivity from slope spectra averaged spatially within bins $25 \mathrm{~m}$ high $\times 400 \mathrm{~m}$ wide, and then smoothing the resulting data spatially with a $1 \times 1$ boxcar. Here, the overall pattern of relatively high dissipation at depths of $\sim 550-600 \mathrm{~m}$ and again at 700 $750 \mathrm{~m}$ (cf. Fig. 20a) is visible, but horizontal variability in this pattern is also evident. The dominant feature is a 50- to 100 -m-thick zone of relatively high diffusivity just above the seafloor, especially at depths near $700 \mathrm{~m}$, where $K_{\rho}$ is as high as $3 \times 10^{-4} \mathrm{~m}^{2} \mathrm{~s}^{-1}$. This zone appears in the seismic image as a region of highly disrupted seismic reflections, suggesting that disruption of fine structure in seismic images may be a useful marker of increased turbulence. 

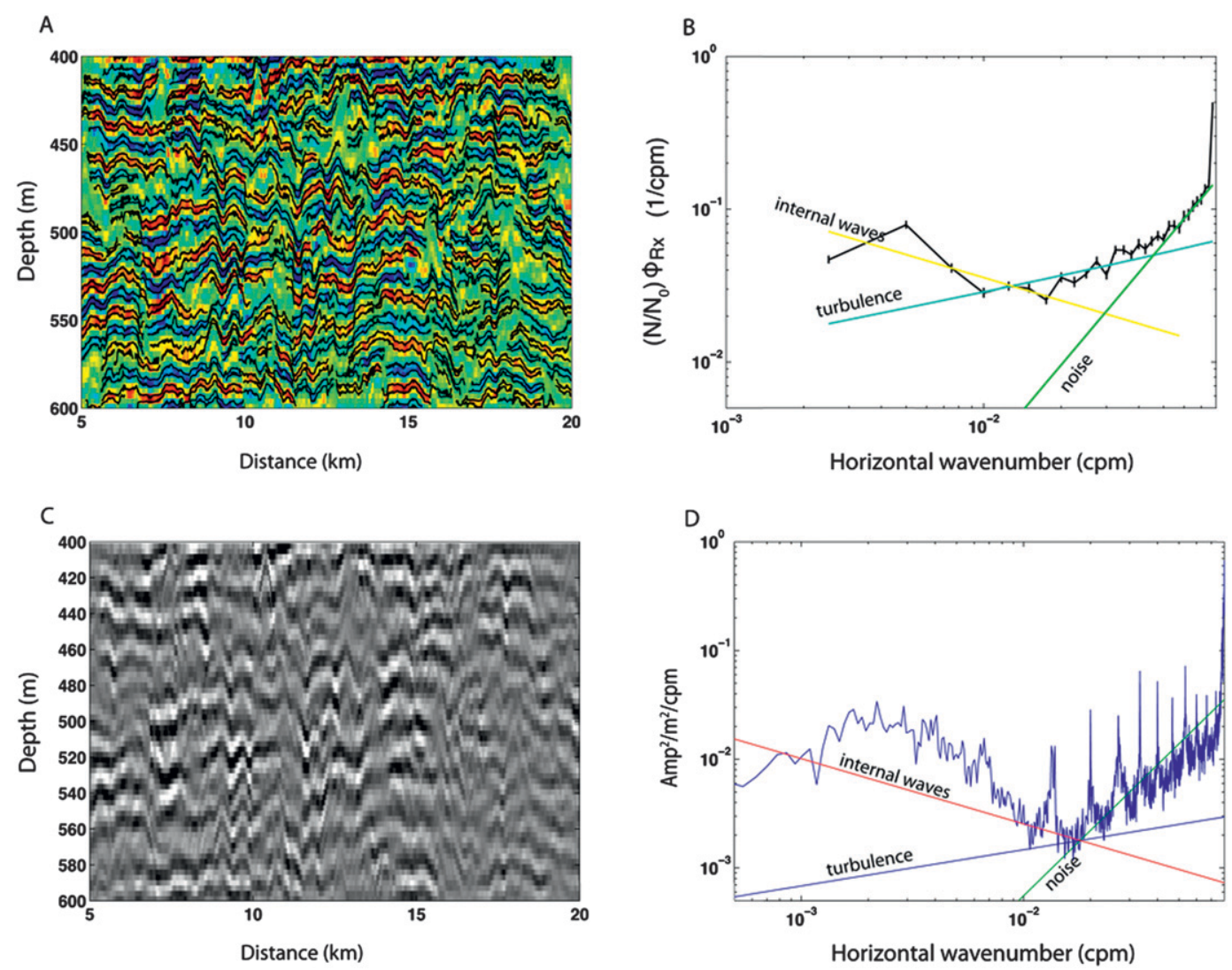

FIG. 21. (a) Tracked reflections, (b) reflector slope spectra $($ NFFT $=64)$, (c) seismic data, and (d) direct data transform of a seismic section acquired in the Gulf of California. Spectrum from tracked reflections shows an apparent (but false) turbulence subrange at middle values of $k_{x}$. The false turbulence slope is caused by spectral leakage from harmonic noise peaks [see (d)], where it is clear that no turbulence subrange is captured in the seismic image; harmonic noise peaks overprint subranges dominated by internal waves and random noise. Spectral leakage from these noise peaks creates a false turbulence slope in (b), thus highlighting the importance of calculating slope spectra directly from seismic data before tracking reflectors.

\section{Discussion}

We have shown that, under the right circumstances, seismic imaging can provide reliable, quantitative estimates of turbulence dissipation in the ocean. Both synthetic modeling and comparisons to in situ estimates from vertical shear support the notion that seismic images can detect and quantify turbulence dissipation. This opens up exciting new possibilities for characterizing the dynamics of turbulence over large swaths of the ocean.

There are, however, several caveats and pitfalls that must be kept in mind when attempting to produce reliable slope spectra from seismic reflections. In particular, both random and $k_{x}$ harmonic noise is present in seismic images and must be analyzed (and suppressed) before deciding whether a particular dataset is appropriate for producing slope spectra. Random noise is easily identifiable because of its clear $k_{x}^{2}$ slope in data transforms
(Fig. 10). Harmonic noise is less familiar and more insidious if it is not identified. In extreme cases, spectral leakage from harmonic noise peaks can produce reflector slope spectra that mimic the expected $k_{x}{ }^{1 / 3}$ slope of the turbulence subrange. An example of this is given in Fig. 21, which shows slope spectra [number of points in the fast Fourier transform (NFFT) $=64]$ from a dataset acquired in the Gulf of California in 2002 aboard the R/V Ewing. Here, the reflector slope spectra (Fig. 21b) produce a subrange that could easily be interpreted as turbulence, but the data transform (Fig. 21d) reveals that this section contains no information about turbulence. Rather, strong harmonic noise peaks overprint subranges dominated by internal waves $\left(k_{x}<0.017 \mathrm{cpm}\right)$ and random noise $\left(k_{x}>0.017 \mathrm{cpm}\right)$. In the reflector slope spectrum, the relatively short Fourier transform length (64) used causes spectral leakage from the harmonic noise peaks that can mimic a turbulence slope and thus produce highly misleading results. When calculating 
$k_{x}$ spectra of seismic oceanography data, it is clearly crucial to consider both random and harmonic noise. Results presented without explicit reporting of the severity of those noise sources, and without accompanying data transforms, should be treated with caution.

The results presented here provide a basis for recommendations about seismic acquisition parameters that are most likely to provide images most amenable to turbulence dissipation estimates. The ideal dataset has high $\mathrm{S} / \mathrm{N}$ values (up to frequencies of at least $80 \mathrm{~Hz}$ ) but minimal contamination from harmonic noise. Increasing $\mathrm{S} / \mathrm{N}$ requires large source arrays to generate strong signal levels, and high fold to facilitate stacking, which can be accomplished by increasing the number of channels (i.e., longer streamer) and/or decreasing the shot spacing. Decreasing shot spacing $\Delta_{s}$ is especially desirable for minimizing harmonic noise, as the noise peaks occur at integer multiples of $1 / \Delta_{s}$. However, when the seafloor and uppermost subseafloor geology is unusually "hard" (high reflection coefficient), decreasing shot spacing can, paradoxically, decrease the $\mathrm{S} / \mathrm{N}$. This is because a major source of "random" noise in seismic oceanography data comes from reverberations within the water column from previous shots. Thus, the increased fold that is gained when going from, say, a shot spacing of 50 down to $25 \mathrm{~m}$ might be swamped by the increased previousshot noise in some environments. The choice of shot spacing will depend on the environment: continental margins covered by soft sediments are amenable to small shot spacings (e.g., $25 \mathrm{~m}$ ), while midocean environments will likely require larger (100-150 m) shot spacings and consequent attention to suppressing harmonic shot noise.

Our work represents a first step in understanding how reliably seismic images can provide turbulence estimates, but much remains to be done. In particular, future studies would benefit both from high-resolution models of ocean dynamics (including patchy turbulence) and from more realistic seismic simulations. More sophisticated and standardized fitting algorithms of the observed slope spectra to the model, also incorporating a realistic noise spectrum, such as the maximum likelihood spectral fitting method to the Batchelor form devised by Ruddick et al. (2000), can increase the accuracy of the inferred diffusivity and the confidence intervals. Seismic oceanography generally, and these methods in particular, would benefit from improved understanding of the causes, time scales, and slope spectral signatures of fine structure in the ocean. For example, under what circumstances does fine structure follow isopycnals? Do both permanent and "reversible" fine structure provide reliable slope spectra, and over what time scales? Most of all, improved confidence in these methods requires further (and better) comparisons between seismic imaging and in situ measurements, including horizontally towed data and microstructure measurements.

\section{Conclusions and recommended workflow}

This study shows that seismic images can provide accurate estimates of turbulence dissipation, out to horizontal wavenumbers of about $0.04 \mathrm{cpm}$, corresponding to horizontal wavelengths as small as $25 \mathrm{~m}$. Comparison of seismically derived turbulence estimates to coincident, simultaneous, in situ estimates from vertical shear corroborate the accuracy of reflector slope spectra and the estimates of turbulence dissipation derived from them. However, producing reliable slope spectra from seismic images of the water column requires recognizing and suppressing two sources of noise: random noise and harmonic noise in the $k_{x}$ domain related to the shot spacing during seismic acquisition. A quick and necessary test of the suitability of a particular seismic dataset to slope spectral analysis is the calculation of slope spectra directly from the seismic data; both random and harmonic noise become evident in such plots.

We summarize a recommended workflow for producing reliable slope spectra from seismic images below:

1) Careful processing through migration. Seismic data must be processed through poststack migration, with careful attention to stacking velocities and sources of noise. Migration is necessary, as unmigrated data have false reflector slopes and thus will not have reliable slope spectra, especially at high $k_{x}$.

2) Calculation of slope spectra directly from seismic data. Before calculating slope spectra from tracked reflectors, it is critical to assess the suitability of each dataset by calculating slope spectra directly from seismic data (Figs. 5b and 6b), as described in section $3 c$. We note that noise characteristics can vary even on a single seismic section because of changes in sea state, bottom characteristics (which can affect previousshot multiple strength), reflector strength, and acquisition parameters; thus, one portion of a seismic line may be suitable for slope spectral analysis (e.g., Fig. 5), while another may not (Fig. 10).

3) Signal-to-noise analysis and random noise suppression. Random noise can be suppressed by carefully selecting filter bands to maximize the signal-to-noise ratio; we found that a band of $30-80 \mathrm{~Hz}$ works well. Each dataset should be analyzed by passband (e.g., Fig. 9 and Table 1) to determine optimal filter bands. Signal-to-noise ratios should be calculated as in Eq. (3) and section 3d; passbands with an $\mathrm{S} / \mathrm{N}$ ratio less than about 4 are unlikely to be suitable. 
4) Suppression of harmonic noise. Shot-generated harmonic noise contaminates all seismic sections (Fig. 11) and must be eliminated by a targeted filter as described in section 3e and shown in Figs. 12. Data acquired with unusually large shot separations will be severely contaminated and may produce false "turbulence" slopes in tracked reflector spectra because of spectral leakage (Fig. 21).

5) Automated reflector tracking. Hand-tracked reflectors are unlikely to produce reliable slope spectra; we recommend automated tracking by contouring the 0.6 value of $\cos (\phi)$, where $\phi$ is the instantaneous phase angle from the Hilbert transform, as described in section $3 \mathrm{f}$.

6) Calculation of reflector slope spectra. Horizontal slope spectra can be calculated from tracked reflectors using the method of KM07, substituting reflector displacements $R_{x}$ for isopycnal displacements $\zeta_{x}$. The effect of varying NFFT on dissipation estimates should be explored, as in Fig. 19.

Acknowledgments. We thank W. Fortin and E. Wilson for numerous discussions and data processing. We thank the captain and crew of the R/V Marcus Langseth, D. Eakin, and K. McIntosh (chief scientist, MGL0908) for data acquisition. The comments of two anonymous reviewers greatly improved the manuscript. This work was funded by NSF Grants 0452744, 0405654, and 0648620, and ONR/DEPSCoR Grant DODONR40027.

\section{REFERENCES}

Barnes, A. E., 2007: A tutorial on complex seismic trace analysis. Geophysics, 72, W33-W43.

Biescas, B., V. Sallarès, J. L. Pelegrí, F. Machín, R. Carbonell, G. Buffett, J. J. Dañobeitia, and A. Calahorrano, 2008: Imaging meddy finestructure using multichannel seismic reflection data. Geophys. Res. Lett., 35, L11609, doi:10.1029/ 2008GL033971.

— L. L. Armi, V. Sallarès, and E. Gracia, 2010: Seismic imaging of staircase layers below the Mediterranean Undercurrent. Deep-Sea Res. I, 57, 1345-1353.

Claerbout, J., and S. Fomel, 2006: Image Estimation by Example: Geophysical Soundings Image Construction. Stanford Exploration Project, $308 \mathrm{pp}$.

D'Asaro, E. A., and J. H. Morison, 1992: Internal waves and mixing in the Arctic Ocean. Deep-Sea Res., 39A, S459-S484.

Eakin, D., W. S. Holbrook, and I. Fer, 2011: Seismic reflection imaging of large-amplitude lee waves in the Caribbean Sea. Geophys. Res. Lett., 38, L21601, doi:10.1029/2011GL049157.

Fer, I., P. Nandi, W. S. Holbrook, R. W. Schmitt, and P. Paramo, 2010: Seismic imaging of a thermohaline staircase in the western tropical North Atlantic. Ocean Sci., 6, 621-631.

Fortin, W. F. J., and W. S. Holbrook, 2009: Sound speed requirements for optimal imaging of seismic oceanography data Geophys. Res. Lett., 36, L00D01, doi:10.1029/2009GL038991.
Garrett, C., and W. Munk, 1975: Space-time scales of internal waves: Progress report. J. Geophys. Res., 80, 291-297.

Gazdag, J., 1978: Wave equation migration with the phase-shift method. Geophysics, 43, 1342-1351.

Gregg, M. C., 1989: Scaling turbulent dissipation in the thermocline. J. Geophys. Res., 94 (C7), 9686-9698.

_ 1999: Uncertainties and limitations in measuring $\epsilon$ and $\chi_{\tau}$. J. Atmos. Oceanic Technol., 16, 1483-1490.

— , T. B. Sanford, and P. D. Pinkel, 2003: Reduced mixing from the breaking of internal waves in equatorial waters. Nature, 422, 513-515.

Henyey, F. S., J. Wright, and S. M. Flatte, 1986: Energy and action flow through the internal wave field: An eikonal approach. J. Geophys. Res., 91 (C7), 8487-8495.

Holbrook, W. S., and I. Fer, 2005: Ocean internal wave spectra inferred from seismic reflection transects. Geophys. Res. Lett., 32, L15604, doi:10.1029/2005GL023733.

, P. Páramo, S. Pearse, and R. W. Schmitt, 2003: Thermohaline fine structure in an oceanographic front from seismic reflection profiling. Science, 301, 821-824.

— I. Fer, and R. W. Schmitt, 2009: Images of internal tides near the Norwegian continental slope. Geophys. Res. Lett., 36, L00D10, doi:10.1029/2009GL038909.

Ivey, G. N., K. B. Winters, and J. R. Koseff, 2008: Density stratification, turbulence, but how much mixing? Annu. Rev. Fluid Mech., 40, 169-184.

Katz, E., 1975: Tow spectra from MODE. J. Geophys. Res., 80, 1163-1167.

Klymak, J. M., and J. N. Moum, 2007a: Oceanic isopycnal slope spectra. Part I: Internal waves. J. Phys. Oceanogr., 37, 12151231. and,- 2007b: Oceanic isopycnal slope spectra. Part II: Turbulence. J. Phys. Oceanogr., 37, 1232-1245.

Kosloff, D., and E. Baysal, 1982: Forward modeling by a Fourier method. Geophysics, 47, 1402-1412.

Krahmann, G., P. Brandt, D. Klaeschen, and T. Reston, 2008: Middepth internal wave energy off the Iberian Peninsula estimated from seismic reflection data. J. Geophys. Res., 113, C12016, doi:10.1029/2007JC004678.

Kunze, E., L. K. Rosenfeld, G. S. Carter, and M. C. Gregg, 2002: Internal waves in Monterey Submarine Canyon. J. Phys. Oceanogr., 32, 1890-1913.

, E. Firing, J. M. Hummon, T. K. Chereskin, and A. M. Thurnherr, 2006: Global abyssal mixing inferred from lowered ADCP shear and CTD strain profiles. J. Phys. Oceanogr., 36, 1553-1576.

Ledwell, J. R., A. J. Watson, and C. S. Law, 1993: Evidence for slow mixing across the pycnocline from an open-ocean tracerrelease experiment. Nature, 364, 701-703.

Lee, C. M., E. Kunze, T. B. Sanford, J. D. Nash, M. A. Merrifield, and P. E. Holloway, 2006: Internal tides and turbulence along the 3000-m isobath of the Hawaiian Ridge. J. Phys. Oceanogr., 36, 1165-1183.

Loewenthal, D., L. Lu, R. Robertson, and J. Sherwood, 1976: The wave equation applied to migration. Geophys. Prospect., 24, 380-399.

Lueck, R. G., and T. D. Mudge, 1997: Topographically induced mixing around a shallow seamount. Science, 276, 18311833.

Mirshak, R., M. R. Nedimović, B. J. W. Greenan, B. R. Ruddick, and K. E. Louden, 2010: Coincident reflection images of the Gulf Stream from seismic and hydrographic data. Geophys. Res. Lett., 37, L05602, doi:10.1029/2009GL042359. 
Moum, J. N., D. R. Caldwell, J. D. Nash, and G. D. Gunderson, 2002: Observations of boundary mixing over the continental slope. J. Phys. Oceanogr., 32, 2113-2130.

Munk, W., and C. Wunsch, 1998: Abyssal recipes II: Energetics of tidal and wind mixing. Deep-Sea Res. I, 45, 1977-2010.

Nakamura, Y., T. Noguchi, T. Tsuji, S. Itoh, H. Niino, and T. Matsuoka, 2006: Simultaneous seismic reflection and physical oceanographic observations of oceanic fine structure in the Kuroshio Extension front. Geophys. Res. Lett., 33, L23605, doi:10.1029/2006GL027437.

Nandi, P., W. S. Holbrook, S. Pearse, P. Páramo, and R. W. Schmitt, 2004: Seismic reflection imaging of water mass boundaries in the Norwegian Sea. Geophys. Res. Lett., 31, L23311, doi:10.1029/2004GL021325.

Nash, J. D., M. H. Alford, E. Kunze, K. Martini, and S. Kelly, 2007: Hotspots of deep ocean mixing on the Oregon continental slope. Geophys. Res. Lett., 34, L01605, doi:10.1029/2006GL028170.

Naveira Garabato, A. C., K. L. Polzin, B. A. King, K. J. Heywood, and J. Visbeck, 2004: Widespread intense turbulent mixing in the Southern Ocean. Science, 303, 210-213.

Osborn, T. R., 1980: Estimates of the local-rate of vertical diffusion from dissipation measurements. J. Phys. Oceanogr., 10, 83-89.

Polzin, K. L., J. M. Toole, and R. W. Schmitt, 1995: Finescale parameterizations of turbulent dissipation. J. Phys. Oceanogr., 25, 306-328.

,-- J. R. Ledwell, and R. W. R. W. Schmitt, 1997: Spatial variability of turbulent mixing in the abyssal ocean. Science, 276, 93-96.

, E. Kunze, J. Hummon, and E. Firing, 2002: The finescale response of lowered ADCP velocity profiles. J. Atmos. Oceanic Technol., 19, 205-224.

Ruddick, B., 1992: Intrusive mixing in a Mediterranean salt lens: Intrusion slopes and dynamical mechanisms. J. Phys. Oceanogr., 22, 1274-1285.

_, A. Anis, and K. Thompson, 2000: Maximum likelihood spectral fitting: The Batchelor spectrum. J. Atmos. Oceanic Technol., 17, 1541-1555.

, H. B. Song, C. Z. Dong, and L. Pinheiro, 2009: Water column seismic images as maps of temperature gradient. Oceanography, 22, 192-205.
Rudnick, D. L., and Coauthors, 2003: From tides to mixing along the Hawaiian Ridge. Science, 301, 355-357.

Sallarès, V., B. Biescas, G. Buffett, R. Carbonell, J. J. Dañobeitia, and J. L. Pelegrí, 2009: Relative contribution of temperature and salinity to ocean acoustic reflectivity. Geophys. Res. Lett., 36, L00D06, doi:10.1029/2009GL040187.

Sanford, T. B., R. G. Drever, J. H. Dunlap, and E. A. D'Asaro, 1982: Design, operation and performance of an expendable temperature and velocity profiler (XTVP). University of Washington, Applied Physics Laboratory Tech. Rep. APL-UW 8110, 164 pp.

Sheen, K. L., N. J. White, and R. W. Hobbs, 2009: Estimating mixing rates from seismic images of oceanic structure. Geophys. Res. Lett., 36, L00D04, doi:10.1029/2009GL040106.

Sheriff, R. E., and L. P. Geldart, 1995: Exploration Seismology. Cambridge University Press, 592 pp.

St. Laurent, L., and C. Garrett, 2002: The role of internal tides in mixing the deep ocean. J. Phys. Oceanogr., 32, 28822899.

Toole, J. M., R. W. Schmitt, K. L. Polzin, and E. Kunze, 1997: Nearboundary mixing above the flanks of a midlatitude seamount. J. Geophys. Res., 102 (C1), 947-959.

Vsemirnova, E., R. Hobbs, N. Serra, D. Klaeschen, and E. Quentel, 2009: Estimating internal wave spectra using constrained models of the dynamic ocean. Geophys. Res. Lett., 36, L00D07, doi:10.1029/2009GL039598.

Watson, D. F., 1982: ACORD: Automatic contouring of raw data. Comput. Geosci., 8, 97-101.

Wessel, P., and W. H. F. Smith, 1991: Free software helps map and display data. Eos, Trans. Amer. Geophys. Union, 72, 441446.

Wunsch, C., and R. Ferrari, 2004: Vertical mixing, energy, and the general circulation of the oceans. Annu. Rev. Fluid Mech., 36, 281-314.

Yamashita, M., K. Yokota, Y. Fukao, S. Kodaira, S. Miura, and K. Katsumata, 2011: Seismic reflection imaging of a warm core ring south of Hokkaido. Explor. Geophys., 42, 18-24.

Yilmaz, O., 1987: Seismic Data Processing. Investigations in Geophysics Series, Vol. 2, Society of Exploration Geophysicists, $526 \mathrm{pp}$. 